\title{
Identification of a Rhythmic Firing Pattern in the Enteric Nervous System That Generates Rhythmic Electrical Activity in Smooth Muscle
}

\author{
Nick J. Spencer, ${ }^{1 *}$ (Timothy J. Hibberd, ${ }^{1 *}$ - Lee Travis, ${ }^{1}$ Lukasz Wiklendt, ${ }^{1}$ Marcello Costa, ${ }^{1}$ Hongzhen Hu, ${ }^{2}$ \\ DSimon J. Brookes, ${ }^{1}$ David A. Wattchow, ${ }^{3}$ Phil G. Dinning, ${ }^{1,3}$ Damien J. Keating, ${ }^{1}$ and Julian Sorensen ${ }^{4}$ \\ ${ }^{1}$ College of Medicine and Public Health and Centre for Neuroscience, Flinders University, Adelaide 5042, Australia, ${ }^{2}$ Department of Anesthesiology, The \\ Center for the Study of Itch, Washington University School of Medicine, St. Louis, Missouri 63110, ${ }^{3}$ Discipline of Surgery and Gastroenterology, Flinders \\ Medical Centre, Bedford Park 5042, South Australia, Australia, and ${ }^{4}$ Cyber Sensing and Shaping, Cyber and Electronic Warfare Division, Defence, Science \\ and Technology Group, Edinburgh, South Australia 5111, Australia
}

The enteric nervous system (ENS) contains millions of neurons essential for organization of motor behavior of the intestine. It is well established that the large intestine requires ENS activity to drive propulsive motor behaviors. However, the firing pattern of the ENS underlying propagating neurogenic contractions of the large intestine remains unknown. To identify this, we used high-resolution neuronal imaging with electrophysiology from neighboring smooth muscle. Myoelectric activity underlying propagating neurogenic contractions along murine large intestine [also referred to as colonic migrating motor complexes, (CMMCs)] consisted of prolonged bursts of rhythmic depolarizations at a frequency of $\sim 2 \mathrm{~Hz}$. Temporal coordination of this activity in the smooth muscle over large spatial fields ( $\sim 7 \mathrm{~mm}$, longitudinally) was dependent on the ENS. During quiescent periods between neurogenic contractions, recordings from large populations of enteric neurons, in mice of either sex, revealed ongoing activity. The onset of neurogenic contractions was characterized by the emergence of temporally synchronized activity across large populations of excitatory and inhibitory neurons. This neuronal firing pattern was rhythmic and temporally synchronized across large numbers of ganglia at $\sim 2 \mathrm{~Hz}$. ENS activation preceded smooth muscle depolarization, indicating rhythmic depolarizations in smooth muscle were controlled by firing of enteric neurons. The cyclical emergence of temporally coordinated firing of large populations of enteric neurons represents a unique neural motor pattern outside the CNS. This is the first direct observation of rhythmic firing in the ENS underlying rhythmic electrical depolarizations in smooth muscle. The pattern of neuronal activity we identified underlies the generation of CMMCs.

Key words: colon; colonic migrating motor complex; enteric nervous system; gastrointestinal tract; myenteric plexus; pacemaker cell

\section{Significance Statement}

How the enteric nervous system (ENS) generates neurogenic contractions of smooth muscle in the gastrointestinal (GI) tract has been a long-standing mystery in vertebrates. It is well known that myogenic pacemaker cells exist in the GI tract [called interstitial cells of Cajal (ICCs)] that generate rhythmic myogenic contractions. However, the mechanisms underlying the generation of rhythmic neurogenic contractions of smooth muscle in the GI tract remains unknown. We developed a high-resolution neuronal imaging method with electrophysiology to address this issue. This technique revealed a novel pattern of rhythmic coordinated neuronal firing in the ENS that has never been identified. Rhythmic neuronal firing in the ENS was found to generate rhythmic neurogenic depolarizations in smooth muscle that underlie contraction of the GI tract.

\section{Introduction}

The gastrointestinal (GI) tract contains its own nervous system, known as the enteric nervous system (ENS). A unique feature of

Received Dec. 7, 2017; revised April 30, 2018; accepted May 9, 2018.

Author contributions: N.J.S. wrote the first draft of the paper; N.J.S., S.J.B., D.A.W., P.G.D., D.J.K., and J.S. edited the paper; N.J.S., M.C., and H.H. designed research; T.J.H. and L.T. performed research; T.J.H. contributed unpublished reagents/analytic tools; T.J.H., L.T., L.W., and J.S. analyzed data; N.J.S., T.J.H., M.C., H.H., and J.S. wrote the paper. the ENS is that it contains entire neural circuits comprising sensory neurons, interneurons, and motor neurons, which can be studied more readily than in the CNS. Another unique feature of the ENS is that isolated segments of intestine can independently

This work was supported by Grants to N.J.S. (1067317 and 1127140) from the National Health and Medical Research Council of Australia.

The authors declare no competing financial interests.

*N.J.S. and T.J.H. contributed equally as co-first authors. 


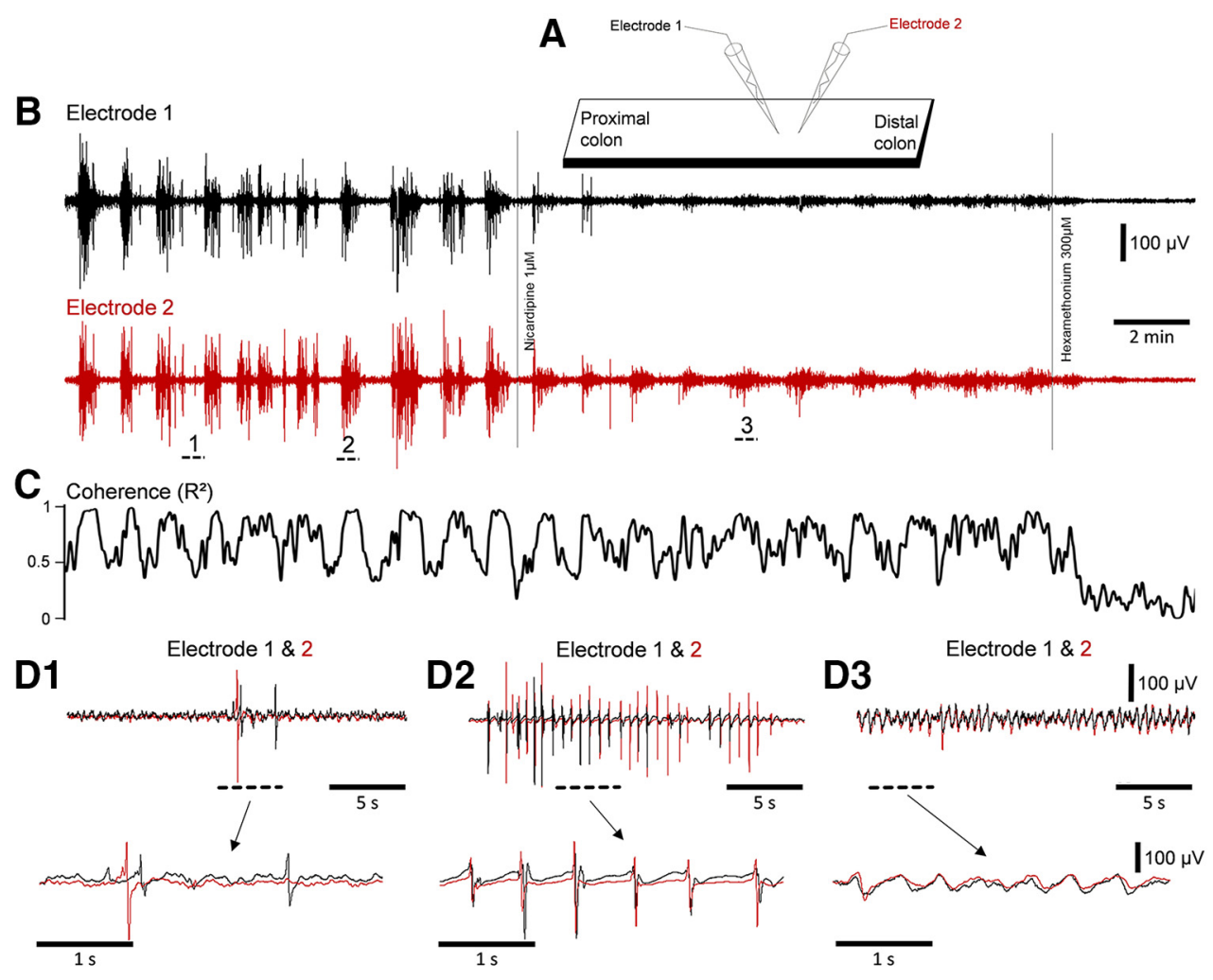

Figure 1. Increased temporal coordination of smooth muscle action potentials during neurogenic spike bursts. $A$, Diagrammatic representation of the full-length colon preparation and dual extracellular recording electrodes separated by $1 \mathrm{~mm}$ in the longitudinal axis. $\boldsymbol{B}$, Simultaneous extracellular recordings made from smooth muscle separated by $1 \mathrm{~mm}$ in the longitudinal axis. The black dotted bar labeled 1 in $B$ indicates a period during the intervals between spike bursts, where action potentials are not spatially and temporally correlated. The period indicated by the bar labeled 2 during a contraction shows a higher level of coherence than during the period at bar 1 (see $\mathbf{C}$ and D1-D3). C, The dynamic changes in coherence in myoelectrical activity over $1 \mathrm{~mm}$ longitudinal distance that occurred between and during the neurogenic spike bursts shown in the recordings in $\boldsymbol{B}$. In nicardipine, muscle action potentials are blocked, but the coherence between bursts of junction potentials still dynamically increases and decreases. In hexamethonium, the dynamic changes in coherence during junction potentials are blocked. D1, D2 and D3, Expanded periods of the recordings indicated by the black bars 1,2 and 3 in $\boldsymbol{B}$. During neurogenic spike bursts (bar 2 ) action potentials become spatially and temporally coordinated at $2 \mathrm{~Hz}$. In Figure 1-1 (available at https://doi.org/10.1523/JNEUROSCI.3489-17.2018.f1-1) hexamethonium is applied before nifedipine. This shows that nicotinic receptor activation in the myenteric plexus is essential for the spatial and temporal coordination of smooth muscle action potentials.

coordinate propulsive movements and propel content without any neural connections to the brain or spinal cord (Brann and Wood, 1976; Costa and Furness, 1976; Brookes et al., 1999; Wood, 2012; Costa et al., 2015; Spencer et al., 2016a).

The ENS consists of millions of neurons that are contained within interconnected micro-ganglia that form two distinct plexuses, known as the myenteric and submucosal plexuses (Furness, 2006; Wood, 2012). Much has been learnt about the different types of neurons within the ENS (Costa et al., 1996; Furness, 2006), particularly in guinea pig ileum (Costa and Brookes, 1994), including their neuronal projections and synaptic inputs and outputs (Bornstein et al., 1994; Furness et al., 1998; Furness, 2006; Mazzuoli and Schemann, 2012; Wood, 2012; MazzuoliWeber and Schemann, 2015). In mammals, the neurons responsible for coordinating motor behavior along the intestine are located in the myenteric plexus and include inhibitory and excitatory motor neurons, ascending and descending interneurons, and a unique population of intrinsic sensory neurons only found in the GI tract (Furness et al., 1998; Mazzuoli and Schemann, 2009, 2012; Mazzuoli-Weber and Schemann, 2015). Despite a detailed knowledge of these neurons, a major unresolved mystery

Correspondence should be addressed to Nick J. Spencer, College of Medicine and Public Health and Centre for Neuroscience, Flinders University, GP0 Box 2100, Adelaide, SA 5042, Australia. E-mail: nicholas.spencer@flinders.edu.au.

DOI:10.1523/JNEUROSCI.3489-17.2018

Copyright $\odot 2018$ the authors $\quad 0270-6474 / 18 / 385508-16 \$ 15.00 / 0$ is how such a large population of neurons behave during the neurogenic motor behaviors that propel content along the bowel (Wood, 2008, 2016; Hu and Spencer, 2018).

It is well known that non-neuronal pacemaker cells exist in the GI tract [known as interstitial cells of Cajal (ICCs)] that generate and control the frequency of myogenic depolarizations in GI smooth muscle cells, known as slow waves (Huizinga et al., 1995, 2014; Dickens et al., 1999). However, rhythmic neurogenic contractions have also been widely recorded in intestinal smooth muscle by many investigators (Brann and Wood, 1976; Spencer and Bywater, 2002; Roberts et al., 2007, 2008; Balasuriya et al., 2016; Spencer et al., 2016a), but their underlying neural activities have been difficult to resolve. Here, we used high resolution neuronal imaging to observe ENS behavior during and between neurogenic contractions in the murine large intestine, that are commonly referred to as colonic migrating motor complexes (CMMCs; Fida et al., 1997). During CMMCs, the ENS was found to generate rhythmic and synchronized firing, involving large populations of cholinergic, nitrergic and CGRP-expressing myenteric neurons.

\section{Materials and Methods}

Tissue dissection. Mice of either sex (C57BL/6J strain; 6-8 weeks old; $18-26 \mathrm{~g}$ ) were killed by isoflurane inhalation as approved by the Animal Welfare Committee of Flinders University (ethics approval 861-13). A ventral midline incision was made and the colorectum, without the ce- 
A

Levels of coherence during synchronised and desynchronised phases of neurogenic activity

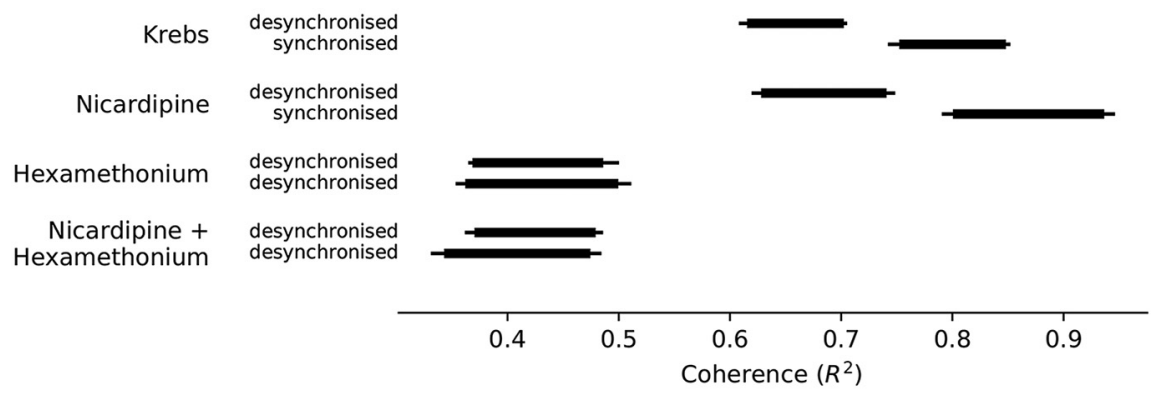

B

Change in coherence from desynchronised to synchronised phases of neurogenic activity

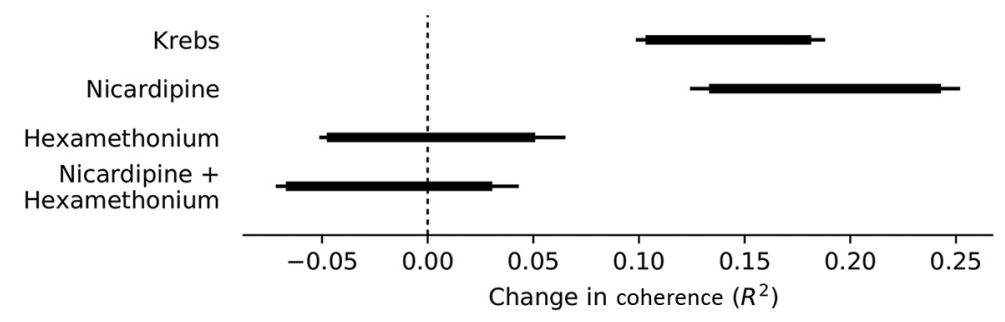

Figure 2. Comparison of the mean coherence between electrodes at a frequency of $\sim 2 \mathrm{~Hz}$ for the desynchronized and synchronized periods associated with the generation of neurogenic spike bursts. Mean coherence posteriors are summarized as $90 \%$ and $95 \%$ highest density intervals depicted with thick and thin lines respectively. $A$, Plot shows the absolute coherence estimates, with changes from desynchronized to synchronized periods during neurogenic spike bursts, shown in the bottom subplot. $\boldsymbol{B}$, There is a clear increase in coherence within the synchronized period compared with the desynchronized period of neurogenic contractions in both Krebs and nicardipine. No difference between the two periods detected in the presence of hexamethonium.

cum $(\sim 50-60 \mathrm{~mm})$ was removed and placed in a Sylgard-lined dissection chamber containing Krebs solution; (in $10^{-3} \mathrm{M}$ concentrations: 118 $\mathrm{NaCl}, 4.7 \mathrm{KCl}, 1 \mathrm{NaH}_{2} \mathrm{PO}_{4}, 25 \mathrm{NaHCO}_{3}, 1.2 \mathrm{MgCl}_{2}, 11$ D-glucose, 2.5 $\mathrm{CaCl}_{2}$, gassed with $95 \% \mathrm{O}_{2}$ and $5 \% \mathrm{CO}_{2}$ ). The gut was flushed of content and cut along the mesenteric border into a flat sheet.

Preparations were mounted in a Sylgard (Dow Corning)-lined recording chamber with micropins (Fine Science Tools; catalog \#26001-10), myenteric plexus uppermost. The entire colon was pinned at a constant circumferential length of $9-10 \mathrm{~mm}$ (slack length $\sim 5 \mathrm{~mm}$ ). This equates to a constant strain of $0.8-1.0$, where strain $=$ elongation $(\mathrm{mm}) /$ original length $(\mathrm{mm})$. The gut was not extended in the longitudinal direction beyond resting length. Preparations were continuously superperfused with Krebs solution $\left(\sim 5 \mathrm{ml} \cdot \mathrm{min}^{-1}, 35^{\circ} \mathrm{C}\right)$. Following equilibration for $30 \mathrm{~min}$, preparations were incubated for $10 \mathrm{~min}$ with Fluo-4-AM (Invitrogen) in solution of 1:1 dimethyl sulfoxide (DMSO) and Cremophor (Sigma-Aldrich)

Calcium imaging. Dynamic changes in cytosolic calcium concentration in myenteric nerve cell bodies were recorded from colonic preparations. Myenteric ganglia were exposed by sharp dissection of longitudinal muscle from the preparation. Images were acquired at $70 \mathrm{~Hz}$ with an electron-multiplied charge coupled device camera (Photometrics Delta Evolve; Roper Scientific). Imaging data were acquired using Imaging Workbench 6.1 software (INDEC BioSystems). Circumferential gut movement was tracked for Figures $4 E$ and $5 D$, using the FIJI "Align slices in stack" tool from the Template Matching plugin, and selecting a box around some continuously illuminated points to track their position over time.

Extracellular and intracellular electrophysiology. The methodology and apparatus for extracellular recordings have been previously described (Hibberd et al., 2017). Extracellular electrodes used for recording from pinned out flat sheet preparations were rigid, whereas electrodes used for recordings from tube preparations had flexible shafts to accom- modate large gut movements. For intracellular recordings, circular muscle cells were impaled with borosilicate micropipettes ( $1 \mathrm{~mm}$ outer diameter, $0.58 \mathrm{~mm}$ inner diameter; Harvard Apparatus) filled with $1 \mathrm{mM} \mathrm{KCl}$, with resistances of 50-120 M $\Omega$. Electrical signals were amplified (Axoclamp 2B amplifier, Molecular Devices), digitized $(20 \mathrm{kHz}$; Powerlab 8/35, ADInstruments), recorded using Chart 7 software (ADInstruments) on a PC. Amplified signals were also visualized on an oscilloscope (TDS-210, Tektronix). Transmural, single pulse electrical stimuli (0.5 ms, 15-30 V; Grass S44 and S1U5) were delivered across the colon using platinum wires mounted on a micromanipulator.

In some experiments, calcium imaging from the myenteric plexus was made at the same time as smooth muscle extracellular or intracellular membrane potential recordings. Preparations were superfused with Krebs solution containing nicardipine $(1 \mu \mathrm{M})$ during intracellular electrophysiological recordings to facilitate microelectrode recordings. Extracellular recordings were performed in regular Krebs solution. All preparations exhibited neurogenic spike bursts, as recently described (Hibberd et al., 2017). All imaged myenteric ganglia were located $15-30 \mathrm{~mm}$ from the terminal rectum. Recordings were performed where neuronal uptake of calcium indicator was considered sufficiently intense and uniform and was free from interference by calcium signals from underlying smooth muscle. Where calcium imaging and smooth muscle extracellular recordings were combined, electrodes were positioned both circumferential and $15 \mathrm{~mm}$ oral to imaged myenteric ganglia. A single circumferential electrode was used in combined calcium imaging and intracellular smooth muscle recordings. In all cases, calcium recordings were restricted to ganglia located within an area $2 \mathrm{~mm}$ oral or aboral to the circumferential recording electrode. Mechanical interference with the water-immersion lens for neuronal imaging precluded electrical recordings directly beneath the imaged ganglia and close to the pinned circumferential edges of preparations (corresponding to the mesenteric border). Thus calcium and electrical recordings were confined to locations between the mesenteric and antimesenteric borders, on opposing sides of the preparation (see Fig. $4 B$ ).

In some experiments, intact tube preparations of colon were used to record smooth muscle electrical activity alone. Electrical activity was simultaneously recorded from the serosal surface by two suction electrodes during propagation of single endogenous, or artificial fecal pellets through the colon.

Immunohistochemical identification of myenteric neurons following calcium imaging. Double-labeling of calcitonin gene-related peptide (CGRP) and nitric oxide synthase (NOS) following calcium imaging was performed as described in (Hibberd et al., 2017, 2018). NOS and choline acetyltransferase (ChAT) double-labeling was performed identically to CGRP/NOS immunolabeling, with the exclusion of organotypic culture in colchicine. Rabbit anti-CGRP (1:2000; Peninsula, catalog \#IHC6006, lot 040826-2; RRID:AB_2314156) and sheep anti-nNOS (1:1000; Emson, catalog \#K205; RRID:AB_2314960) was used donkey anti-rabbit IgG (1:200; Cy3, The Jackson Laboratory, catalog \#711 165 152), and donkey anti-sheep IgG (1:100; Cy5, The Jackson Laboratory, catalog \#713 175 147), respectively. Sheep anti-nNOS (1:1000; Emson, catalog \#K205; RRID:AB_2314960) and rabbit anti-ChAT (1:1000; Schemann/ P3YEB) were used in conjunction with donkey anti-sheep IgG (1:100; Cy5, The Jackson Laboratory, catalog \#713 175 147) and donkey antirabbit IgG (1:200; Cy3, The Jackson Laboratory, catalog \#711 165 152), respectively. 
A

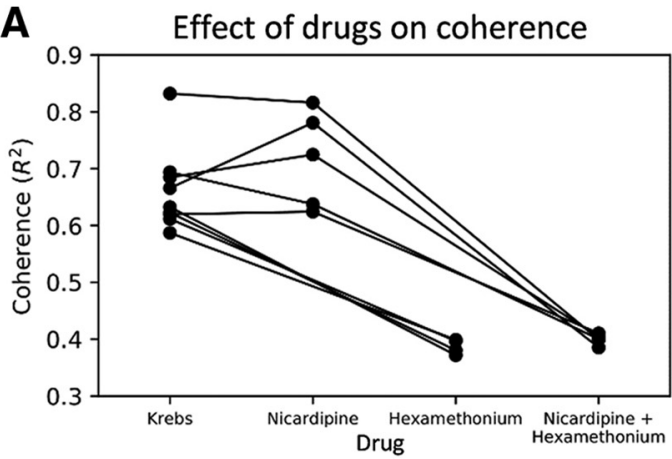

C

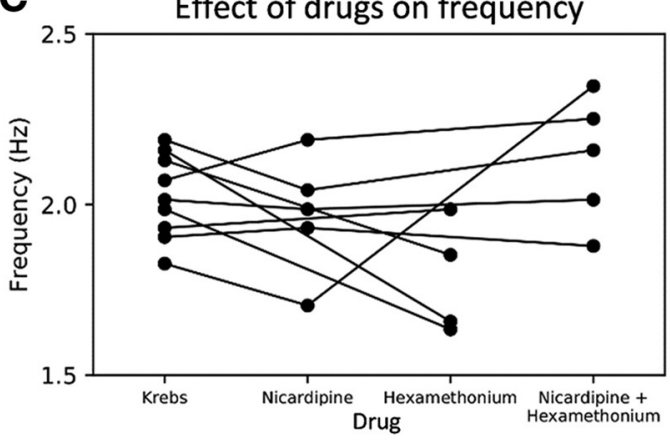

E

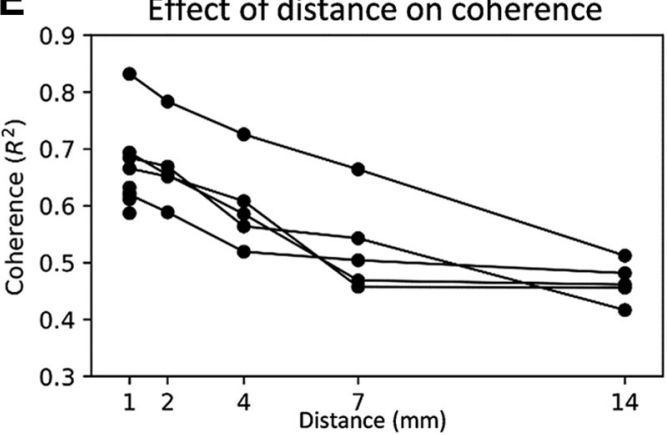

G

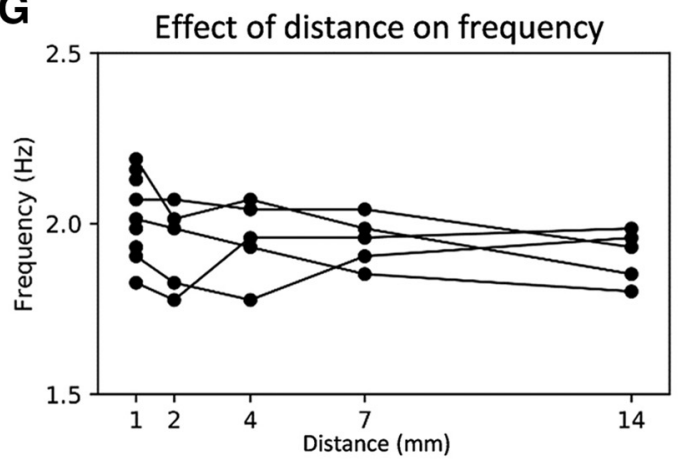

B

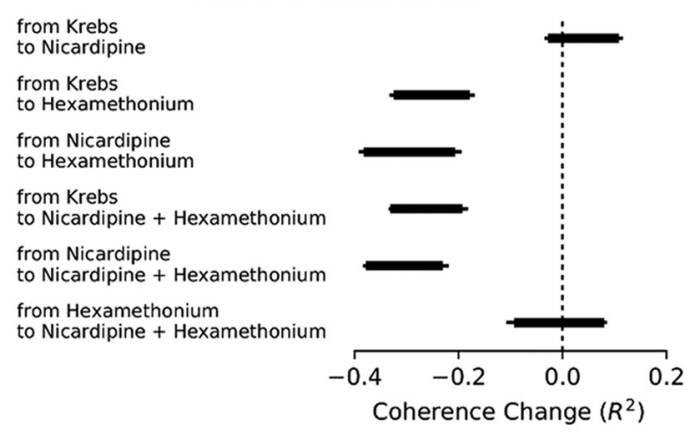

D

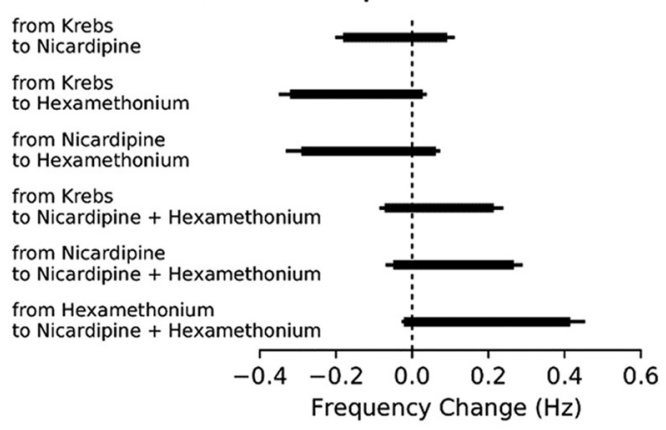

$\mathbf{F}$

Relative coherences

Relative frequencies
Slope of distance effect on coherence

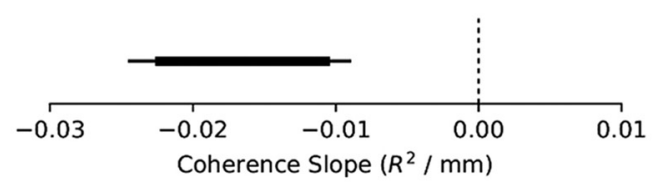

H

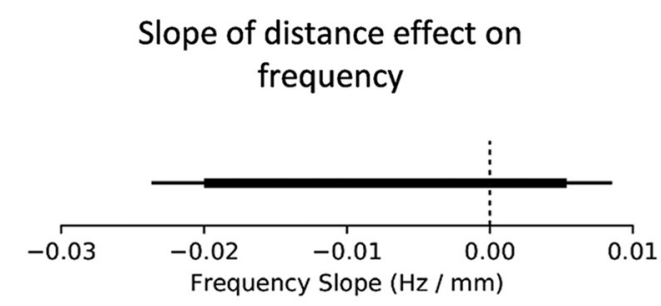

Figure 3. A, The effect of drugs on myoelectric coherence over a distance of $1 \mathrm{~mm}$. B , The associated $90 \%$ and $95 \%$ intervals of the posterior distribution (depicted as thick and thin lines, respectively) for the mean coherence in $\boldsymbol{A}$. Myoelectric coherence in hexamethonium alone and in hexamethonium + nicardipine was significantly reduced compared with Krebs solution or nicardipine alone. There was no significant difference between Krebs solution and nicardipine alone, nor between hexamethonium alone and hexamethonium + nicardipine. $C$, The effect of drugs on the highest-amplitude myoelectric frequency and $(\boldsymbol{D})$ the posterior distribution intervals. No significant differences in frequency occurred between any of the drug combinations. $\boldsymbol{E}$, Myoelectric coherence over space (longitudinal separation of electrodes). $\boldsymbol{F}$, The associated $90 \%$ and $95 \%$ intervals of the posterior distribution of the regression slope in $\boldsymbol{E}$. This demonstrates a significant inverse relationship between myoelectric coherence and longitudinal distance. In comparison, there was no change in the maximum-amplitude myoelectric frequency associated with distance along the gut $(\boldsymbol{G}, \boldsymbol{H})$.

Terminology. The methods presented here refer to synchronized and desynchronized periods, which are characterized in the results section. Briefly, synchronized periods correspond to periods of time during neurogenic spike bursts which are characterized by relatively high spatial coordination of myoelectric activity and coordinated neural activity. De- synchronized periods correspond to time intervals between neurogenic spike bursts and are characterized by relatively low spatial coordination of myoelectric activity and uncoordinated neural activity.

Myoelectric coherence and frequency analysis. Comparison of myoelectric voltage coherence under various conditions was performed with the 

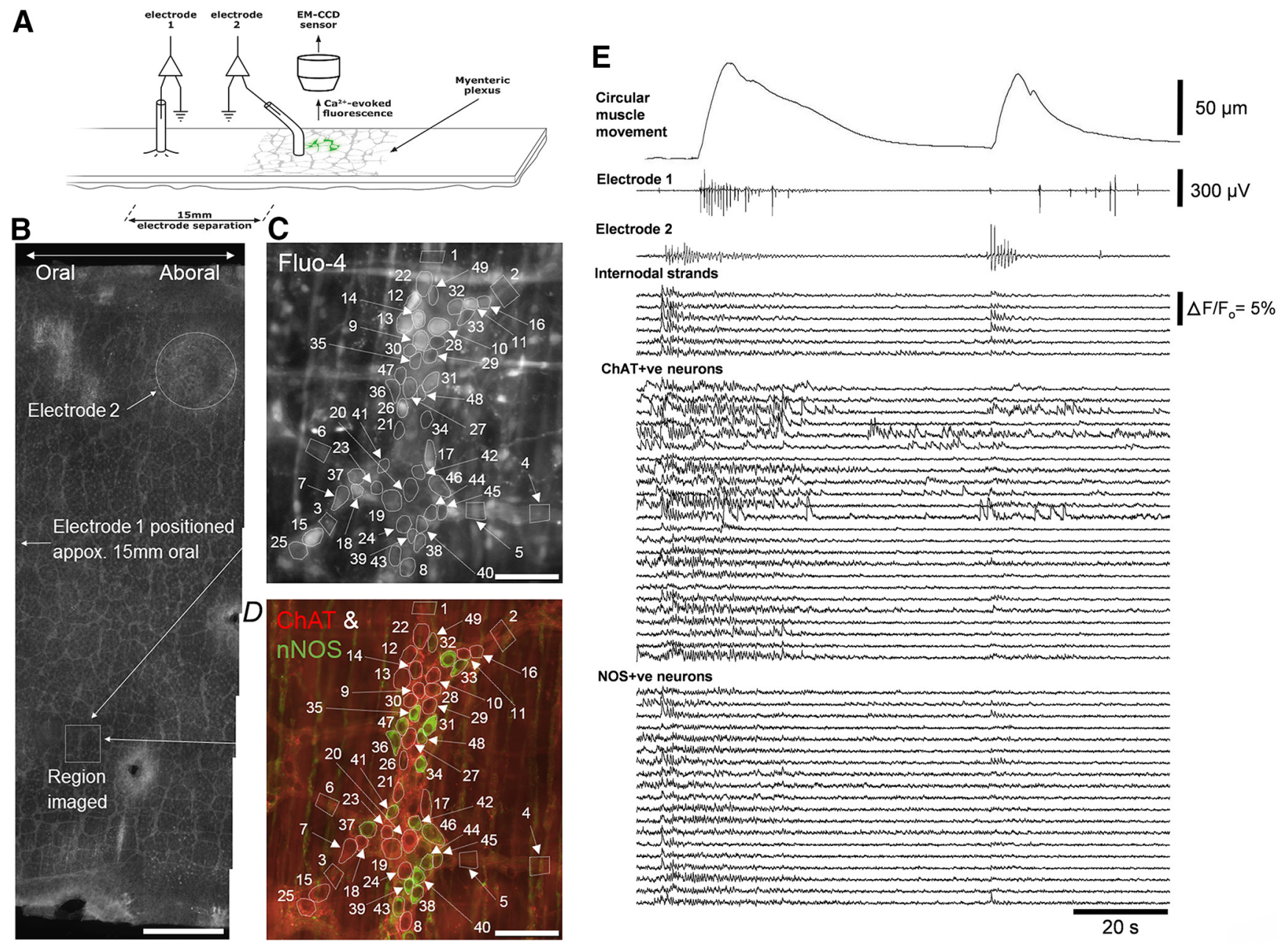

Figure 4. Simultaneous neuronal imaging and smooth muscle electrophysiology during propagating neurogenic spike bursts in whole mouse colon. $A$, Schematic of the location of the neuronal imaging and electrophysiological recordings. Two extracellular recording electrodes were positioned $15 \mathrm{~mm}$ apart along the length of colon. $\boldsymbol{B}$, The full circumference of mouse colon and the location of the myenteric ganglia imaged relative to the position of electrode 2. Calibration, $1 \mathrm{~mm}$. C, Fluo-4 in the live preparation showing the recorded neurons and internodal strands. Calibration, $100 \mu \mathrm{m}$. $D$, shows the neurochemical coding of the region shown in C. Numerous excitatory (ChAT+ve in red) and inhibitory (NOS + ve in green) neurons are shown in neighboring myenteric ganglia. Calibration, $100 \mu \mathrm{m}$. E, shows periodic neurogenic contractions and propagating bursts of muscle action potentials recorded from electrodes 1 and 2 . Before and during the neurogenic contractions, neuronal imaging showed increased activity in internodal strands, ChAT + ve and NOS + ve neurons. Figure 4-1 (available at https://doi.org/10.1523/JNEUROSCI.3489-17.2018.f4-1) shows that synchronized firing of ChAT + ve and NOS + ve neurons was also temporally coordinated with activation of motor nerve axons in the circular muscle and occurred in all detectable nerve cell bodies.

"rstanarm" R package. This allowed us to compute the posterior probability distribution of various parameters of interest, conditioned on the data we had observed.

For comparisons of synchronized and desynchronized periods, a hierarchical model was used for partial pooling of neurogenic spike bursts within recording blocks, and blocks within each preparation (individual animal) and drug. This was given by the linear mixed-effects formula: "coherence $\sim$ (period $\mid$ drug/block $)+($ period $\mid$ preparation/block)" with the default weakly informative priors.

To clarify, a recording block contained on the order of tens of timeintervals. Each interval was considered a single unit of data, and contained the measured coherence over the timespan of that interval as its response value. There were four predictors of the response: (1, period) the classification of the type of period it belongs to, that is, either synchronized or desynchronized; (2, drug) the drug combination the preparation was exposed to at the time of the interval; (3, preparation) an indicator for which animal preparation it belongs to; and (4, block) an indicator for the recording block.

The above formula could be conceptually reduced to "coherence (period | drug)" because we were interested in whether there is a difference in coherence between the synchronized and desynchronized periods, with this difference potentially varying between drugs. However, because we had multiple potentially correlated measurements of coher- ence for each type of period in each recording block, rather than simply averaging the coherences of each type of period in a block, we instead used a nested model "coherence $\sim$ (period $\mid$ drug/block)". This effectively performed such an averaging while also accounting for potential correlations and propagating the information of individual intervals to the overall distribution that would otherwise have been lost with simple averaging. The formula's second term "(period | preparation/block)" accounts for potential random deviations in the coherence of different period types for each animal preparation. Note: each block contained only one type of drug and was a recording for only one of the animals, that is, the drug was not changed within a block, nor were recordings from multiple animals fused into one block. However, there were multiple blocks recorded for each drug and each animal. For comparisons of myoelectric coherence and frequency in the presence of drugs at various electrode separation distances, the formula "measure $\sim$ distance + (distance | preparation)" was used to infer the slope with distance of a measure of either coherence or frequency. The formula "measure $\sim(1 \mid$ drug $)+(1 \mid$ preparation)" was used to infer the intercept over drugs, with the default weakly informative priors.

Signal processing. The wavelet transform and coherences were calculated as in (Torrence and Compo, 1998), using a Morlet wavelet with a nondimensional frequency of $\omega_{0}=6$, for scales corresponding to the frequencies between 0.5 and $8 \mathrm{~Hz}$. Where coherence was used, it was 
A

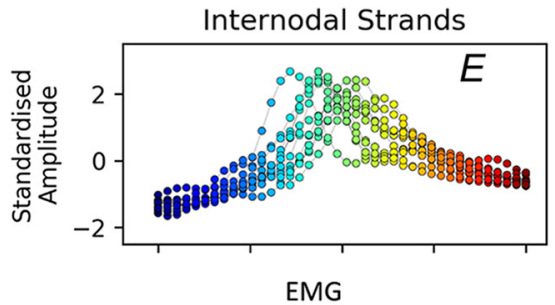

B

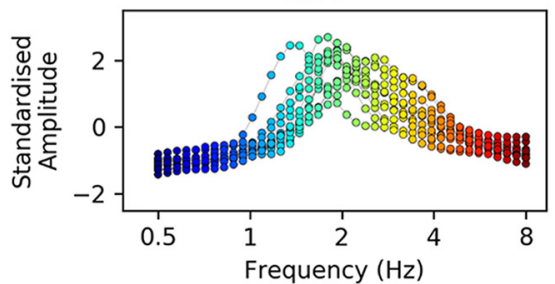

D

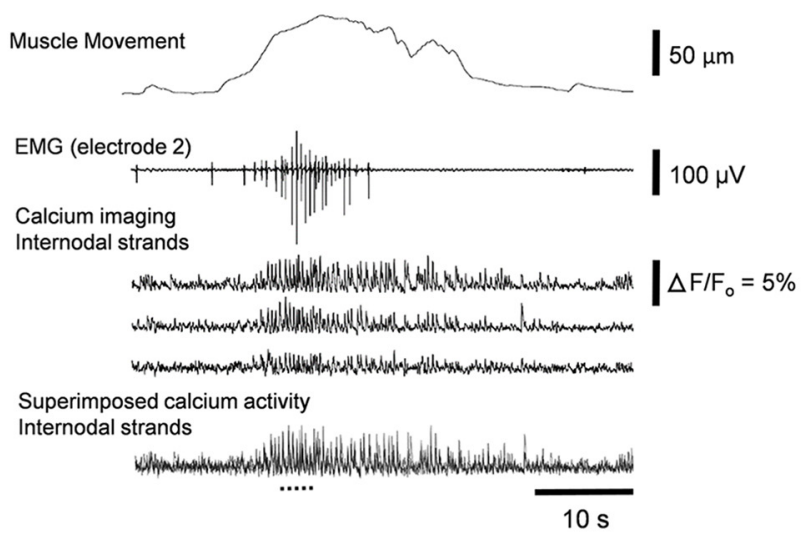

C EMG vs Internodal Strands

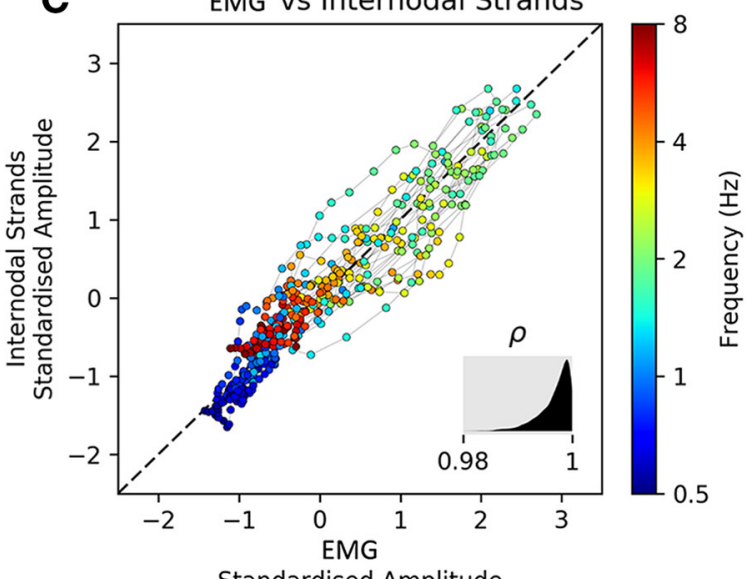

Standardised Amplitude

E

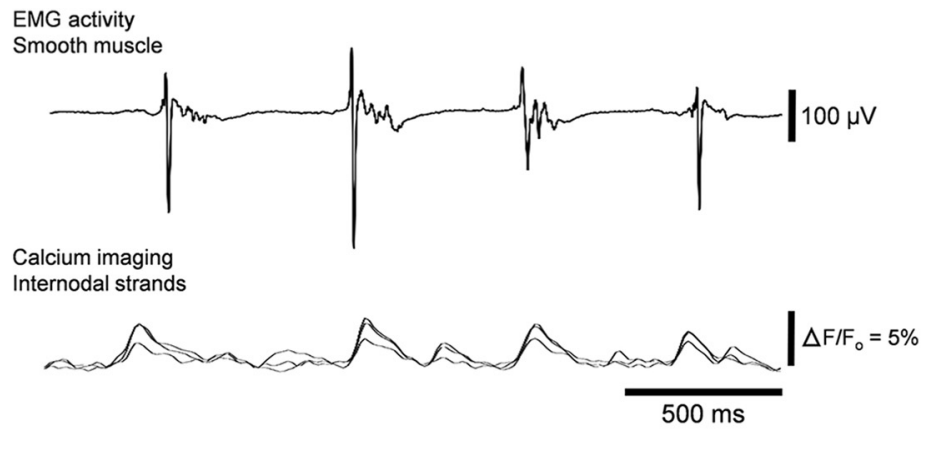

Figure 5. Correlation of electrical activity in smooth muscle (EMG) with neuronal imaging from internodal strands, varying over frequencies. The wavelet transform was calculated for calcium imaging of internodal strands $(\boldsymbol{A})$ and the electrical activity recorded from the smooth muscle $(\boldsymbol{B})$, for 40 scales corresponding to frequencies from 0.5 to $8 \mathrm{~Hz}$. Calcium signals generated in internodal strands were averaged into a single signal. We calculated the correlation of standardized global wavelet spectrum amplitudes over frequencies between the internodal strands and the electrical activity from the smooth muscle. C, The two spectra were correlated with a correlation coefficient of at least 0.98 . Thus the frequencies of calcium transients from myenteric ganglia and the rates of depolarization in smooth muscle were highly correlated. The posterior density of the overall correlation $(\rho)$ is shown in the gray inset at the bottom right. $\boldsymbol{D}$, Example recording of a neurogenic contraction, showing mechanical activity, EMG (from electrode 2, closest to imaged ganglia), and the calcium-evoked fluorescence in internodal strands. Expanded EMG and calcium fluorescence traces corresponding to the underlined region in $\boldsymbol{D}$ are shown in $\boldsymbol{E}$, where the imaging and electrode signals were manually aligned for presentation. Synchronized activation of large populations of myenteric ganglia was resistant to blockade of smooth muscle action potentials (with nicardipine), as demonstrated in Figure 5-1 (available at https://doi.org/10.1523/JNEUROSCI.3489-17.2018.f5-1), but was abolished by hexamethonium.

calculated at the frequency with the maximum amplitude of the global wavelet spectrum (which was usually $\sim 2 \mathrm{~Hz}$ ). A preprocessing step was also performed, where each EMG signal was split into positive and negative voltages, and the wavelet transform was computed separately on the log-positive and log-negative portions of the signal, and then the two were averaged to obtain the final wavelet transform amplitudes. The logarithmic transformation allowed us to reduce the artifactual influence of sharp spikes, due to muscle action potentials, on higher-frequency harmonics.

Methodology for imaging analysis. We have recently described in detail the techniques used here for the analysis of temporal correlation of calcium transients (Sorensen et al., 2017). Briefly, we used four independent analytical techniques to compare the degree of temporal coordination of neuronal activity among populations of myenteric neurons between synchronized and desynchronized periods. Three measures were based on the cross correlogram and cross-correlation functions. These are referred to below as follows:

Measure 1: proportion of cross correlograms maximized close to a time lag of 0 .

Measure 2: proportion of cross-correlations maximized close to a time lag of 0 .

Measure 3: average value of the cross-correlation function at a time lag of 0 .
These pairwise measures tested the one-sided hypothesis that temporal coordination between myenteric neurons increased during neurogenic contractions. A paired permutation test was used to test this hypothesis for each measure. We used a paired test because the measures are calculated using the same neuronal sites during the synchronized and desynchronized periods. To avoid the potential correlation between measure values that share a common neuron, we applied the permutation test to subsets of values, where subsets were chosen such that each site was associated with only one value.

In addition to pairwise comparisons, a Measure 4 was used to assess coordination of calcium transients arising from all neurons in the field of view (1-3 myenteric ganglia in the present study). This requires an analysis of the coincident activity patterns, which are patterns of calcium transients that are aligned within a certain time tolerance $(\sim 86 \mathrm{~ms})$. In the present study, the distribution of the number of sites engaged in a coincident pattern of calcium transients was heavily skewed toward the right (i.e., more of the patterns of calcium transients involved a smaller number of sites). As such, simply comparing the mean number of sites engaged in coincident firing pattern was inappropriate. We therefore compared the maximum number of sites engaged in a coincident pattern of calcium transients. More specifically, for both the synchronized and desynchronized periods, we identified and compared the coincident calcium transients engaging the maximum number of sites. 

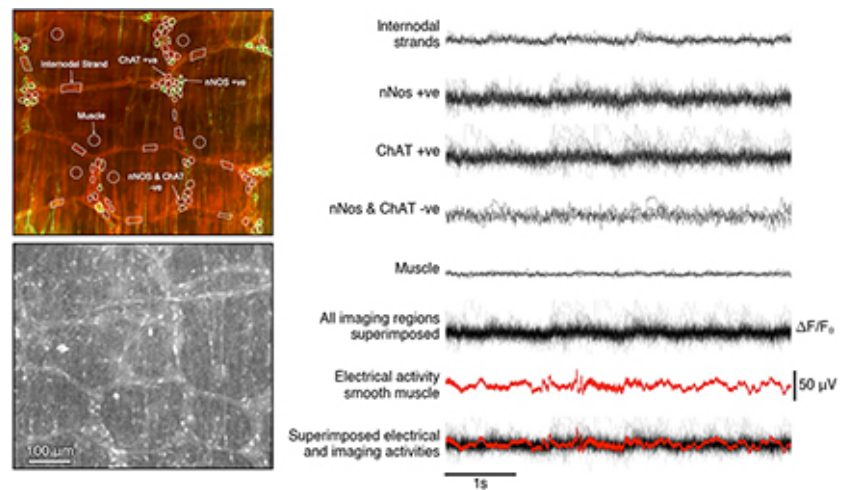

Movie 1. Simultaneous neuronal imaging with electrophysiology from colonic smooth muscle during desynchronized neuronal activity. The top left panel shows the neurochemical content of the neurons from which calcium recordings were made. NOS + ve neurons are shown in green; ChAT + ve neurons shown in red. The lower left panel shows the same area as the top left panel in the live preparation, loaded with

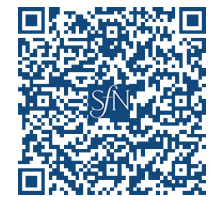
calcium indicator, Fluo-4. White circles indicate the individual regions from which the calcium recordings were taken. The smooth muscle was paralyzed with nicardipine (1 $\mu \mathrm{M})$, so that junction potentials could be recorded at the same time as neuronal imaging. Here, the right hand side shows simultaneous calcium and smooth muscle electrical recordings in an interval between neurogenic contractions. This shows a relative absence of coordinated neuronal activity and smooth muscle junction potentials.

Table 1. Average calcium discharge rates across various neurochemical classes of myenteric neurons, internodal strands, and varicosities during neurogenic contractions (synchronized periods), between neurogenic contractions (desynchronized periods), and in hexamethonium

\begin{tabular}{|c|c|c|c|c|c|c|}
\hline Structure & Neurochemistry & $\begin{array}{l}\text { Sync, Hz } \\
\text { (mean } \pm \text { SD) }\end{array}$ & $\begin{array}{l}\text { Desync, } \mathrm{Hz} \\
(\text { mean } \pm \mathrm{SD})\end{array}$ & $\begin{array}{l}\text { Hexamethonium, } \\
\mathrm{Hz} \text { (mean } \pm S D \text { ) }\end{array}$ & Count & $n$ \\
\hline Cell body & CGRP + /NOS - & $1.5 \pm 0.5$ & $1.0 \pm 0.4$ & $0.00 \pm 0.00$ & 10 & 5 \\
\hline Cell body & CGRP $+/$ NOS + & 1.5 & 1.2 & - & 1 & 5 \\
\hline Cell body & CGRP-/NOS + & $1.6 \pm 0.3$ & $0.8 \pm 0.4$ & $0.01 \pm 0.04$ & 64 & 5 \\
\hline Cell body & CGRP-/NOS- & $1.8 \pm 0.4$ & $0.8 \pm 0.5$ & $0.01 \pm 0.04$ & 104 & 5 \\
\hline Cell body & ChAT+/NOS- & $1.8 \pm 0.6$ & $0.6 \pm 0.5$ & $0.00 \pm 0.00$ & 194 & 7 \\
\hline Cell body & ChAT+/NOS + & 0.7 & 1.2 & 0.00 & 1 & 7 \\
\hline Cell body & ChAT-/NOS + & $1.9 \pm 0.3$ & $0.8 \pm 0.6$ & $0.00 \pm 0.00$ & 136 & 7 \\
\hline Cell body & ChAT-/NOS- & $1.9 \pm 0.5$ & $0.8 \pm 0.6$ & $0.00 \pm 0.00$ & 20 & 7 \\
\hline $\begin{array}{l}\text { Internodal } \\
\text { strands }\end{array}$ & Uncoded & $1.9 \pm 0.3$ & $0.9 \pm 0.5$ & $0.01 \pm 0.06$ & 105 & 12 \\
\hline Varicosities & Uncoded & $1.9 \pm 0.4$ & $1.0 \pm 0.5$ & $0.00 \pm 0.02$ & 84 & 12 \\
\hline All & & $1.8 \pm 0.4$ & $0.8 \pm 0.5$ & $0.00 \pm 0.03$ & 719 & 12 \\
\hline
\end{tabular}

Methodologies for calculating analysis Measures 1, 2, 3, and 4. We outline the steps taken to calculate Measures 1, 2, 3 and 4, and give some relevant parameters required. A more detailed description is presented by Sorensen et al. (2017). In the present study, the gradient method (Sorensen et al., 2017) was applied to bandpass filtered data for calcium transient detection.

The cross correlogram histograms demonstrate the spike timing differences between two spike trains. Here, the bin size used was six samples $(\sim 86 \mathrm{~ms})$. To perform cross-correlation, unstable baselines were removed with a median filter, using a Hanning window, of length 60 samples $(\sim 860 \mathrm{~ms})$; the median filtered data then had a low pass filter applied, where the cutoff value of the filter was $9 \mathrm{~Hz}$. For cross correlogram analyses, the maximum tolerance for alignment of calcium recordings was set at six samples $(\sim 86 \mathrm{~ms})$ to be consistent with the bin size used; for the cross-correlation this tolerance was four samples ( $\sim 57 \mathrm{~ms})$. For analysis of coincident activity patterns, perfect alignment in a coincident pattern of calcium transients is not expected. However, we do expect the calcium transients to fall within a bin of a small size, where the size of the bin is a fraction of the average time between calcium transient
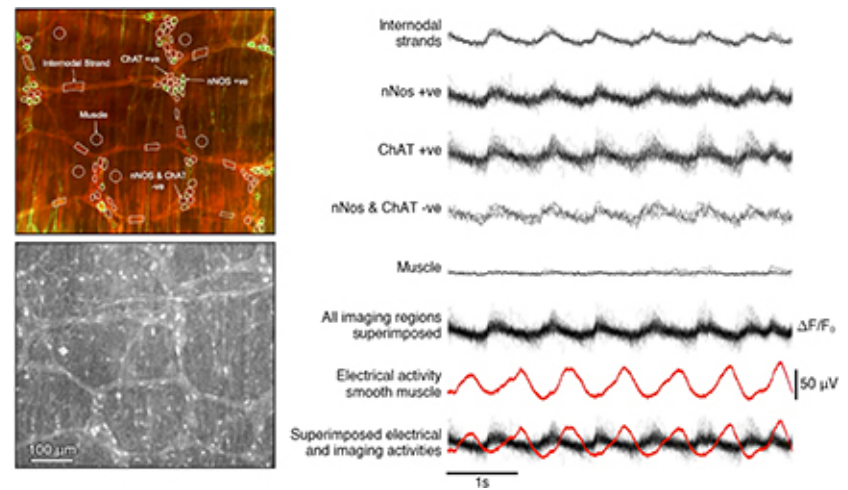

Movie 2. Simultaneous neuronal imaging with electrophysiology from colonic smooth muscle during synchronized neuronal activity. Recordings here correspond to the same sources shown in Movie 1. Here, neural and smooth muscle activity was captured during a neurogenic spike burst. As in Movie 1, the top left panel shows the neurochemical content of the neurons from which calcium recordings were made. NOS + ve neurons are shown in green; ChAT + ve neurons shown in red. The lower left panel shows the same area as the top left panel in the live preparation, loaded with calcium indicator, Fluo-4. White circles indicate the individual regions from which the calcium recordings were taken. In nicardipine $(1 \mu \mathrm{m})$, junction potentials were recorded during neuronal imaging. Simultaneous calcium recordings from all indicated structures during a neurogenic spike burst are shown on the right. Temporal coordination of calcium transients occurred between internodal strands and all neurochemical classes of myenteric neurons. Excitatory junction potentials in the smooth muscle occurred $\sim 100 \mathrm{~ms}$ after the coordinated neuronal activation.

events. In our analysis this bin size was six samples ( $~ 86 \mathrm{~ms})$. For consistency we used the same bin size in unitary event analysis.

Test statistics used for Measures 1, 2 and 3. The major challenge in any hypothesis-testing scenario is finding a test statistic whose assumptions are met by the data; the mathematics behind any statistical test has assumptions regarding the data. Although marginal distribution assumptions can often be quite relaxed, properties regarding the correlation between samples are usually not. For Measures 1, 2 and 3 we identified two potential sources of correlation in the data, the major source of correlation being the potential correlation of the measures between the synchronized and desynchronized periods. To explain further, suppose that we have $M$ neurons resulting in $N=M(M-1) / 2$ cross-correlation/ cross correlograms; let $\left\{Q_{n}\right\}_{n=1}^{N}$ and $\left\{R_{n}\right\}_{n=1}^{N}$ be the collection of boolean observations, taking value 0 or 1 (where a value of 1 denotes the maximization of the cross-correlation/cross correlogram close to a time lag of 0 ), for all pairs during the synchronized $(Q)$ and desynchronized $(R)$ periods. Now, for each $n$, the observations $Q_{n}$ and $R_{n}$ may be correlated as they are measured from the same neuronal imaging sites. Hence a paired statistical test must be used to test the following one-sided hypotheses:

$$
\begin{aligned}
& H_{0}: \frac{1}{N} \sum_{i=1}^{N} Q_{i} \leq \frac{1}{N} \sum_{i=1}^{N} R_{i}, \\
& H_{1}: \frac{1}{N} \sum_{i=1}^{N} Q_{i}>\frac{1}{N} \sum_{i=1}^{N} R_{i} .
\end{aligned}
$$

Similarly, for Measure 3, we require a paired test to compare the values of cross-correlation at time lag 0 between the synchronized and desynchronized periods.

Another correlation that may exist is between measurements that share a common neuron. To elaborate further, suppose that $Q_{i}$ is associated with neurons $\mathrm{A}$ and $\mathrm{B}$ (i.e., $Q_{i}$ denotes whether the cross correlogram for neurons $A$ and $B$ has been maximized close to a time lag of 0 ), and $Q_{j}$ is associated with neurons $B$ and C. The measures $Q_{i}$ and $Q_{j}$ may be correlated due to the common neuron $\mathrm{B}$. We addressed this issue by subsampling $\left\{Q_{n}\right\}_{n=1}^{N}$ and $\left\{R_{n}\right\}_{n=1}^{N}$ repeatedly, forming a collection of 

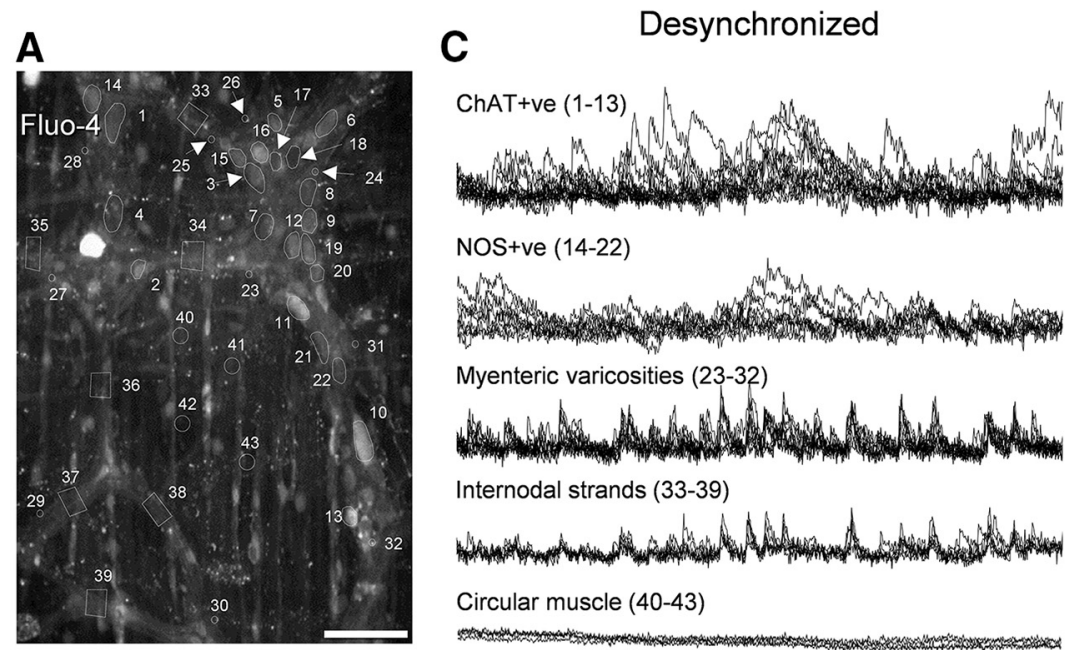

\section{E}

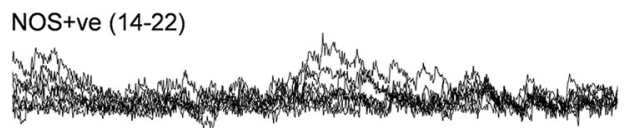

Myenteric varicosities (23-32)

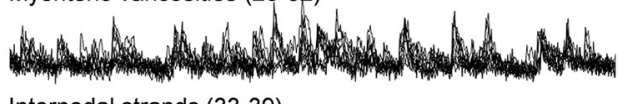

Internodal strands (33-39)

ChAT+ve (1-13)

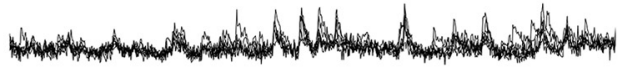

Circular muscle (40-43)

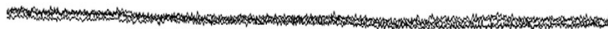

B

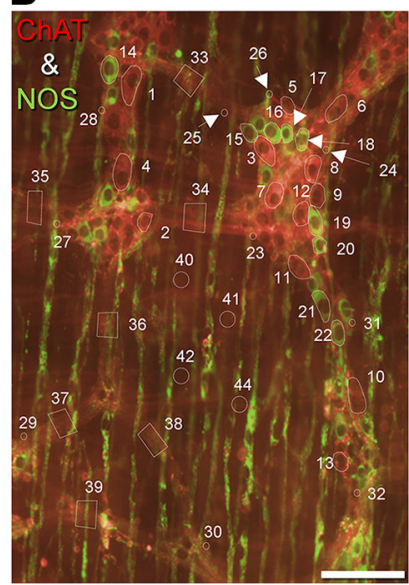

D

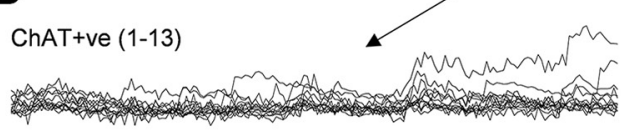

NOS+ve (14-22)

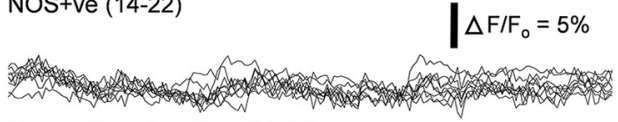

Myenteric varicosities (23-32)

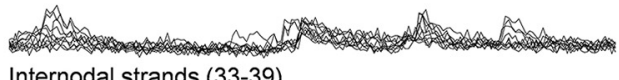

Internodal strands (33-39)

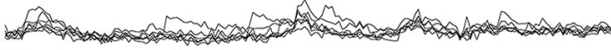

Circular muscle (40-43)

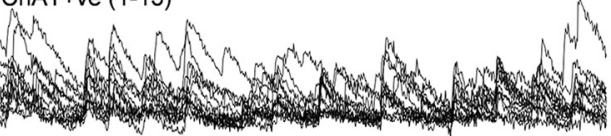

NOS+ve (14-22)

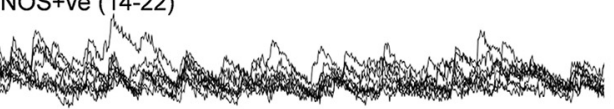

Myenteric varicosities (23-32)

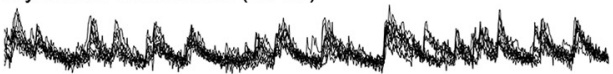

Internodal strands (33-39)

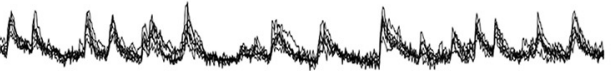

Circular muscle (40-43)

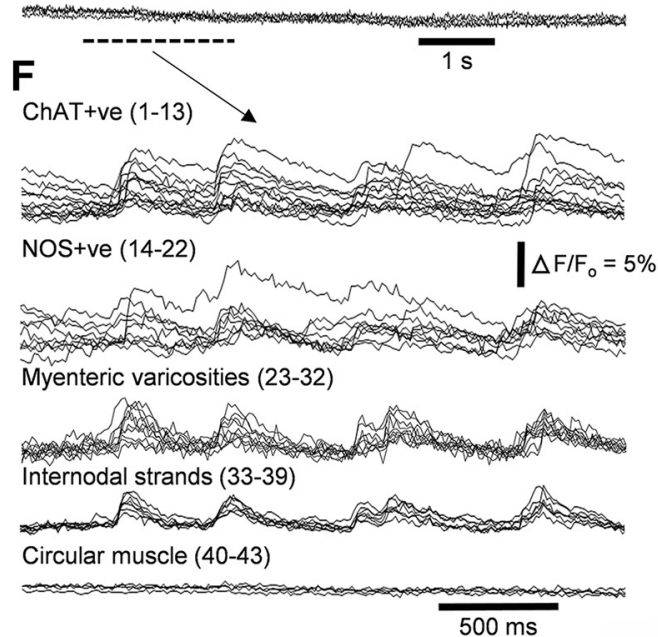

Figure 6. Two distinct periods of synchronized and desynchronized neuronal activity occurred in the myenteric plexus during propagating neurogenic contractions. $\boldsymbol{A}$, The structures (dotted outlines) from which calcium recordings were taken. These include ChAT+ ve nerve cell bodies (numbered 1-13), N0S + ve nerve cell bodies (14-22), myenteric varicosities (22-32), internodal strands (33-39), and sections of circular muscle (40-43). Recordings were made in the presence of nicardipine (1 $\mu \mathrm{m})$ to paralyze the smooth muscle. $\boldsymbol{B}$, The neurochemical coding of the imaging region shown in $\boldsymbol{A}$. Scale bar, $100 \mu \mathrm{m}$. C, During the intervals between neurogenic contractions, there was weak temporal coordination of calcium transients from cholinergic and nitrergic neurons, myenteric varicosities and internodal strands. Scale bar, $100 \mu \mathrm{m}$. $\boldsymbol{D}$, The period corresponding to the dashed bar in $\boldsymbol{C}$. Relatively weak temporal coordination between the neuronal structures occurred. $\boldsymbol{E}$, Activity from the same sources in $\boldsymbol{A}$, but with temporal coordination between the different classes of nerve cell bodies, internodal strands, and varicosities. $\boldsymbol{F}$, Expanded region of the recording period represented by the dotted line in $\boldsymbol{E}$. Comparison of recordings $\boldsymbol{E}$ and $\boldsymbol{F}$ shows the changes in coordination of neuronal firing between desynchronized and synchronized states. Of particular interest was the observation that during synchronized neuronal activity, CGRP + ve myenteric neurons also became temporally activated with all the ChAT + ve and NOS + ve neurons, as shown in Figure 6-1 (available at https://doi.org/10.1523/JNEUROSCI.3489-17.2018.f6-1).

subsets $\left\{\left\{Q_{l}\right\}_{l \in L_{k}}\right\}_{k=1}^{10000}$ and $\left\{\left\{R_{l}\right\}_{l \in L_{k}}\right\}_{k=1}^{10000}$ such that each $L_{\mathrm{k}}$ has the following properties:

(1) $\left|L_{\mathrm{k}}\right|=\lfloor\mathrm{M} / 2\rfloor$

(2) Each neuron can only be associated with one $Q_{l}$ for ${ }_{1} \in L_{k}$.

The second property ensures that for each $L_{\mathrm{k}}$, the measurements $\left\{Q_{l}\right\}_{l \in L_{k}}$ cannot be correlated due to a commonly shared neuron between the measurements (i.e., no $Q_{l} \in\left\{Q_{l}\right\}_{l \in L k}$ share a common neuron). For each subset we performed the paired permutation tests found in (Higgins, 2003), and calculated the percentage of subsets such that there was statistical difference between measures for the synchronized and desynchronized periods. For each subset we applied 10,000 permutations.

Statistics. Differences in datasets were considered statistically significant if $p<0.05$. Results are expressed as mean $\pm \mathrm{SD}$, unless stated otherwise. Lower case " $n$ " always indicates the number of animals used in a set of experiments. Bayesian analysis was performed using Stan (Carpenter et al., 2017) and rstanarm (Stan Development Team, 2017). Four formal statistical tests were undertaken, all of which were one sided: Measures 1, 2 and 3 (see Methodology for imaging analysis) used paired permutation tests found by Higgins (2003) and the statistical significance of a particular coincident activity pattern was tested using the test statistic used in unitary event analysis. An $\alpha$-value of 0.05 was used in all cases.

Drugs. The following stock solutions of drugs were made: hexamethonium chloride in water $\left(10^{-1} \mathrm{M}\right.$; Sigma-Aldrich, H2138); nicardipine hydrochloride in DMSO (10 ${ }^{-1}$ M; Sigma-Aldrich, N7510). Drugs were kept refrigerated and diluted on the day of the experiment to working concentrations in Krebs solution.

\section{Results}

Coordination of smooth muscle electrical activity

Extracellular electrical recordings were made of the myoelectric activity of the colonic smooth muscle layers from two recording sites along the mid to distal region of isolated whole mouse colon $(n=7$; Fig. $1 A)$. Cyclical bursts of smooth muscle action potentials were recorded (Fig. $1 B$ ), which have been previously described as "neurogenic spike bursts" (Hibberd et al., 2017). Neurogenic spike bursts lasted for $32.7 \pm 2.1 \mathrm{~s}$, with intervals of $1.5 \pm 0.5 \min (n=7$; Fig. 1$)$. A striking feature was that during 

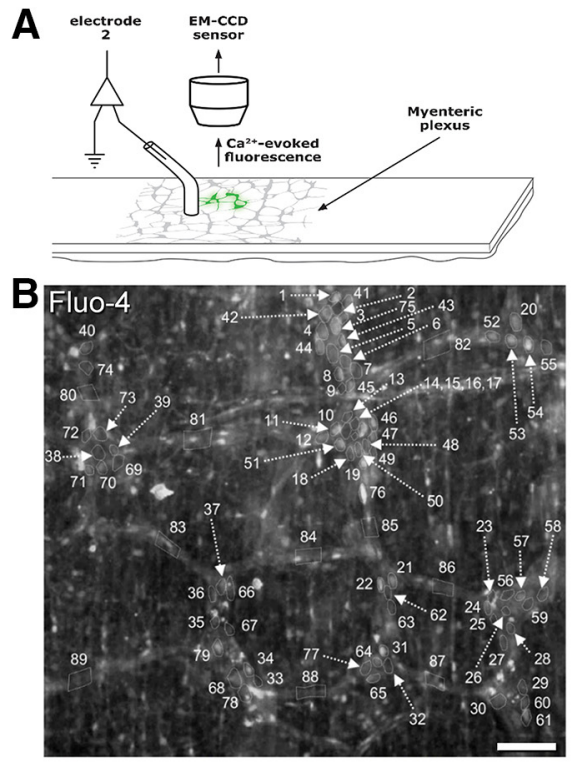

C

Desynchronized

ChAT+ve (1-40)

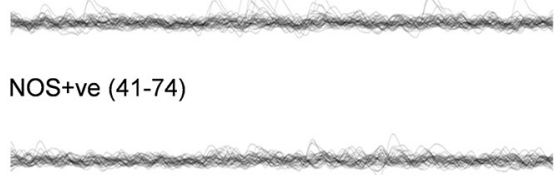

NOS-ve/ChAT-ve (75-79)

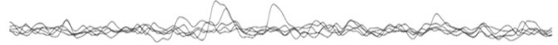

Internodal strands (80-89)

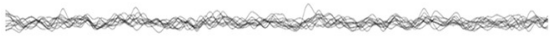

Superimposed imaging (1-89)

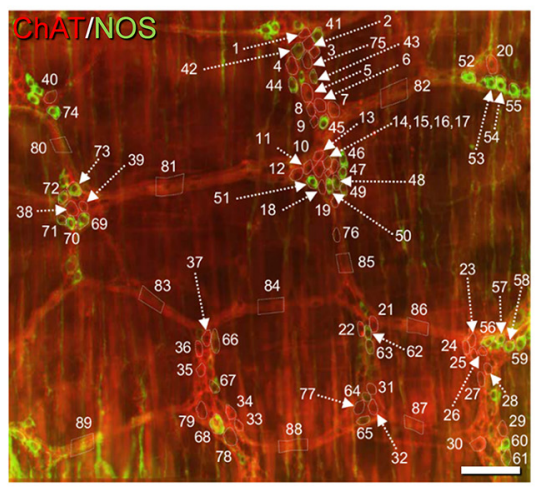

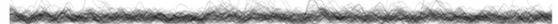

Superimposed imaging (1-89; ave.)

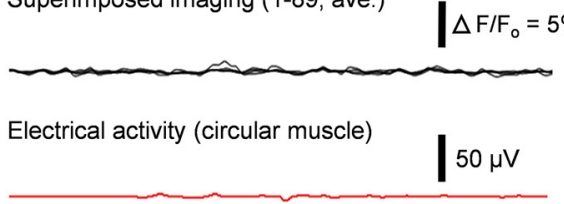

Electrical activity circular muscle + superimposed imaging (1-89)
D Synchronized

ChAT+ve (1-40)

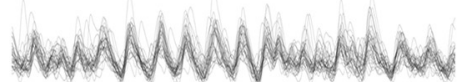

NOS+ve (41-74)

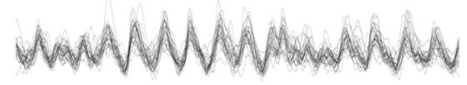

NOS-ve/ChAT-ve (75-79)

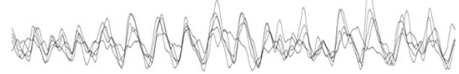

Internodal strands (80-89)

undublum

Superimposed imaging (1-89)

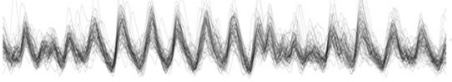

Superimposed imaging (1-89; ave.)

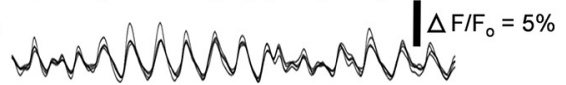

Electrical activity (circular muscle)

NWANAMNAMNANA

Electrical activity circular muscle

+ superimposed imaging (1-89)

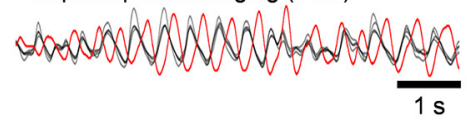

Figure 7. Simultaneous electrical recording from smooth muscle and calcium imaging from neurons during synchronized neuronal activity along the colon. $\boldsymbol{A}$, Schematic diagram of the preparation used with a single extracellular recording electrode and closely apposed calcium imaging site on myenteric ganglia. $\boldsymbol{B}$, Top micrograph shows the neuronal imaging region from myenteric plexus. Bottom, The neurochemical coding of the neurons shown in the top. Calibration, $100 \mu \mathrm{m}$. C, Neuronal activity from different neurochemical classes of neurons and the smooth muscle electrical activity during desynchronized activity. Imaging shows relative inactivity from NOS + ve and ChAT + ve neurons and internodal strands from the locations outlined and numbered in $\boldsymbol{B}$. The calcium and electrical recordings during this period shows the lack of synchronized neuronal activity and relatively quiescent smooth muscle electrical activity. $\boldsymbol{D}$, activity in the same set of structures indicated in $\boldsymbol{B}$ and $\boldsymbol{C}$. when discrete calcium transients are synchronized with each other. Recordings $\boldsymbol{C}$ and $\boldsymbol{D}$ were made in the presence of nicardipine $(1 \mu \mathrm{m})$ and were taken $\sim 90 \mathrm{~s}$ apart. When electrical recordings were made from the muscle, rhythmic depolarizations occurred in the smooth muscle at the same rate as calcium transients in the myenteric ganglia. The depolarizations were activated out of phase with respect to enteric neurons ( $\boldsymbol{D}$, bottommost superimposed traces). To demonstrate the delay in activation of smooth muscle following activation of enteric nerves, Figure 7-1 (available at https://doi.org/10.1523/JNEUROSCI.3489-17.2018.f7-1) shows the effects of single pulse transmural electrical stimulation.

neurogenic spike bursts, smooth muscle action potentials occurred at a remarkably regular rate $(2.0 \pm 0.04 \mathrm{~Hz}$; Fig. 1D2). Recordings of neurogenic spike bursts from two sites $1 \mathrm{~mm}$ apart revealed myoelectric activity was highly coordinated during neurogenic spike bursts (measured as coherence; Fig. 1C). That is, extracellular potentials at both recording sites showed high linear correlation. However, lower correlations occurred during periods between neurogenic spike bursts (Fig. 1C). Thus, myoelectric coherence over a distance of 1 $\mathrm{mm}$ oscillated in parallel with the occurrence of neurogenic spike bursts. Coherence peaks occurred during neurogenic spike bursts and troughs occurred in the periods between them (Fig. 1C). We refer to these periods as synchronized and desynchronized, respectively. The difference in coherence between synchronized and desynchronized periods are shown in Figure $2 A$ and is readily visualized in raw myoelectric voltage traces by comparing electrodes 1 and 2 in Figure 1, D1 and D2. In the presence of nicardipine $(1 \mu \mathrm{M})$, neurogenic bursts of junction potentials occurred at $\sim 2 \mathrm{~Hz}$, without smooth muscle action
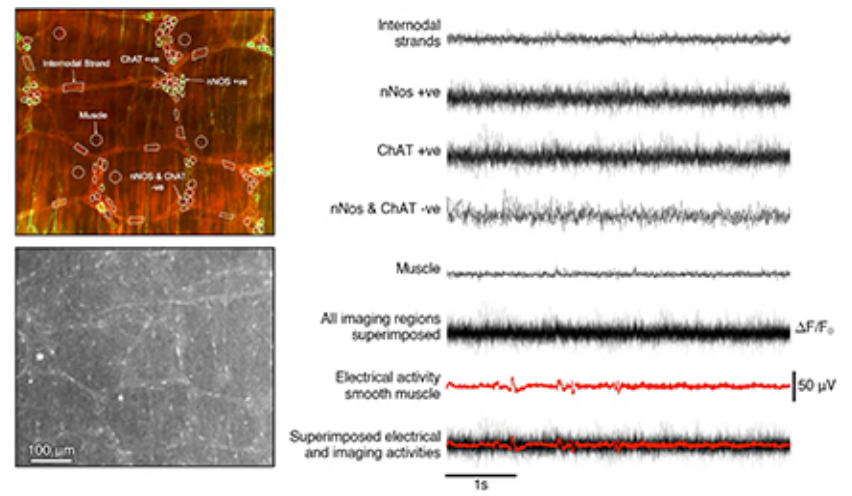

Movie 3. Simultaneous neuronal imaging with electrophysiology from colonic smooth muscle in the presence of hexamethonium. In the presence of hexamethonium, the coordinated neuronal activity and synchronized calcium transients between ganglia (as shown in Movie 2) was abolished. Movies 1-3 were all obtained from the same locations and from the same preparation.

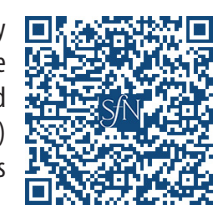


A

\section{Neuronal calcium transient frequency (overall; $\mathbf{n}=\mathbf{1 5}$ )}

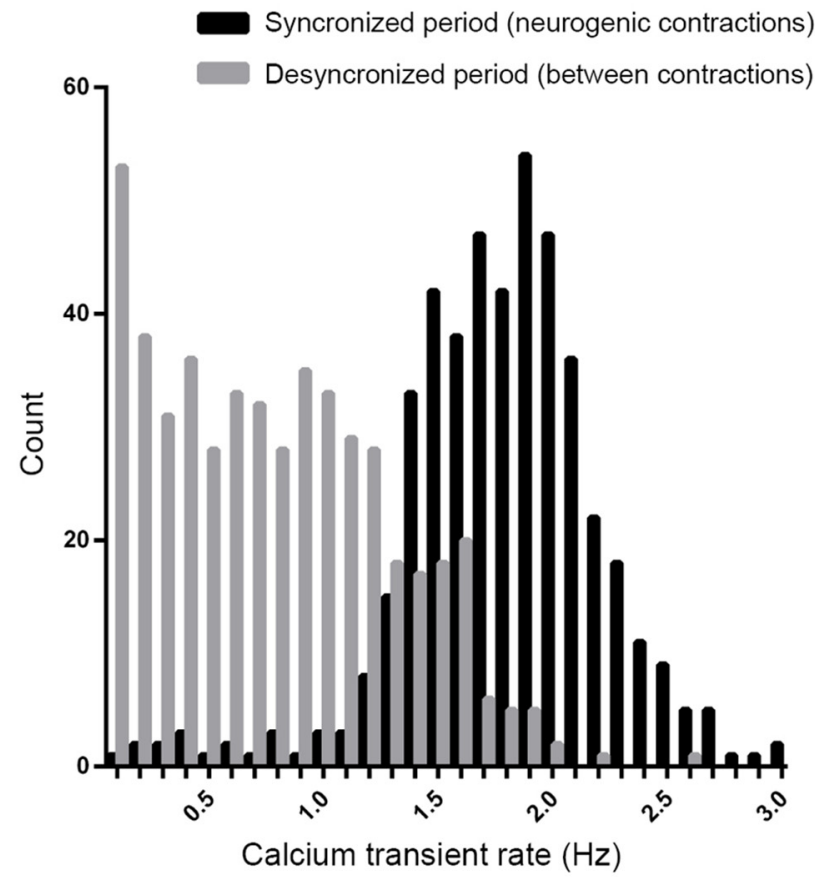

C

\section{Calcium transient frequency in nerve cell bodies, synchronized periods}

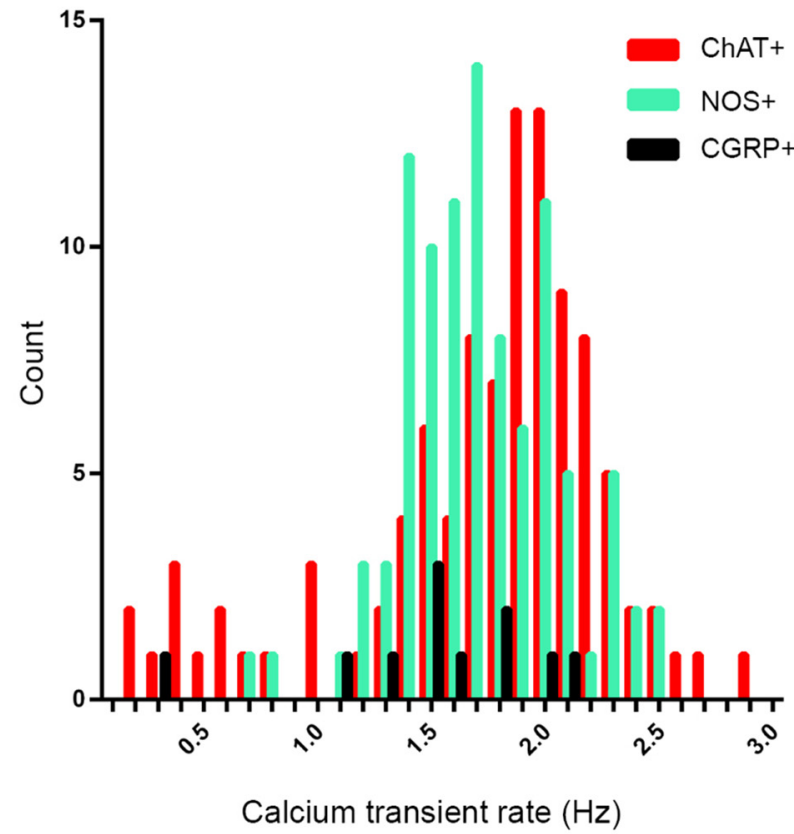

B

\section{Neuronal calcium transient frequency (nerve cell bodies; $n=15$ )}

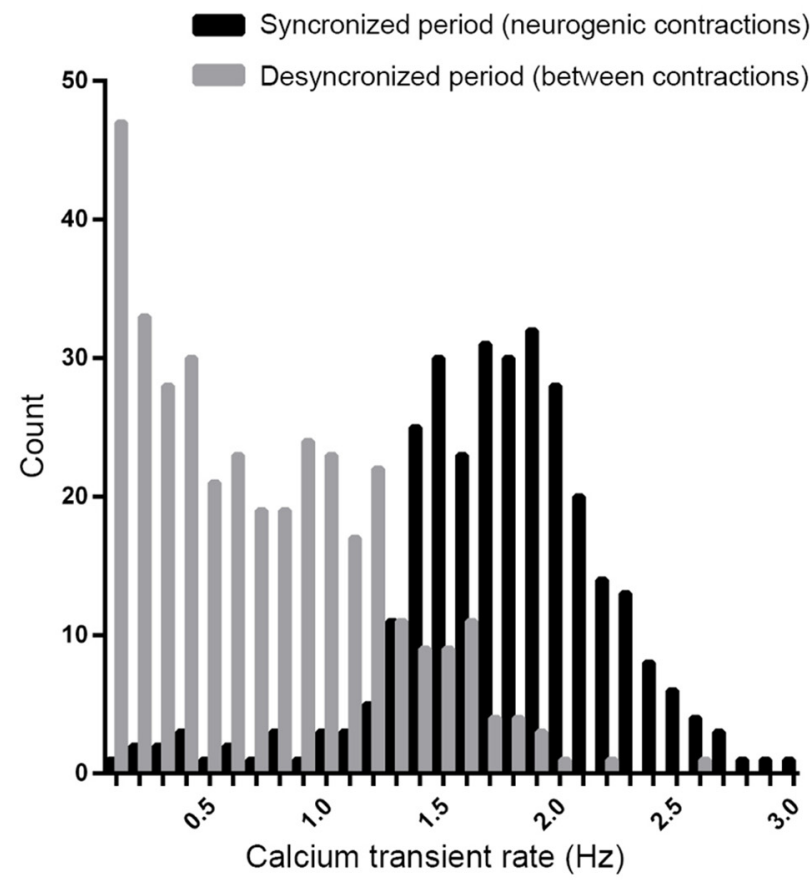

D

\section{Calcium transient frequency in nerve cell bodies, desynchronized periods}

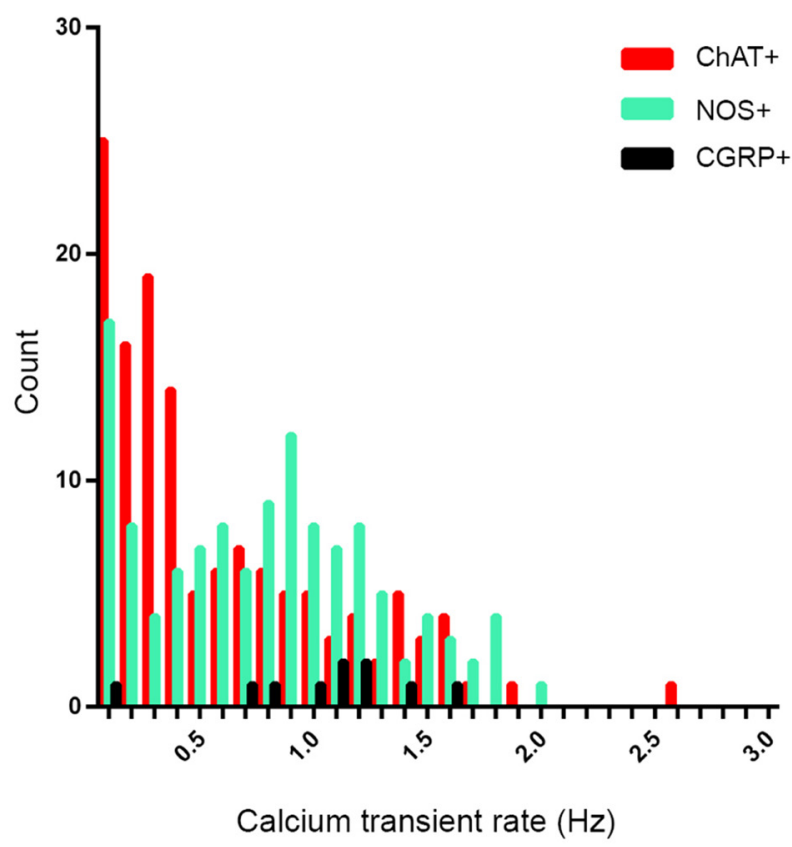

Figure 8. Distribution of neuronal calcium transient frequencies during and between neurogenic contractions. $\boldsymbol{A}$, Frequencies of calcium transients in all myenteric nerve cell bodies, internodal strands and varicosities in synchronized and desynchronized periods $(n=12)$. Ongoing calcium transients occurred in desynchronized periods. $\boldsymbol{B}$, Frequencies of calcium transients in all myenteric nerve cell bodies in synchronized and desynchronized periods $(n=12)$. C, Frequencies of calcium transients in neurochemically-characterized myenteric nerve cell bodies during synchronized periods. $\boldsymbol{D}$, Frequencies of calcium transients in neurochemically-characterized myenteric nerve cell bodies during desynchronized periods.

potentials $(n=5)$ and the relationship between neurogenic bursts and myoelectric coherence persisted (Fig. 1B-D, Fig. 1-1 available at https://doi.org/10.1523/JNEUROSCI.3489-17.2018. f1-1, Fig. 2A,B). The nicotinic receptor antagonist hexametho- nium (300 $\mu \mathrm{M} ; n=5$ ), abolished neurogenic spike bursts (Fig. 1-1 available at https://doi.org/10.1523/JNEUROSCI.3489-17.2018. f1-1) and significantly reduced coherence between the two recording electrodes (Fig. 2). Hexamethonium also abolished the $\sim 2 \mathrm{~Hz}$ bursts 
A

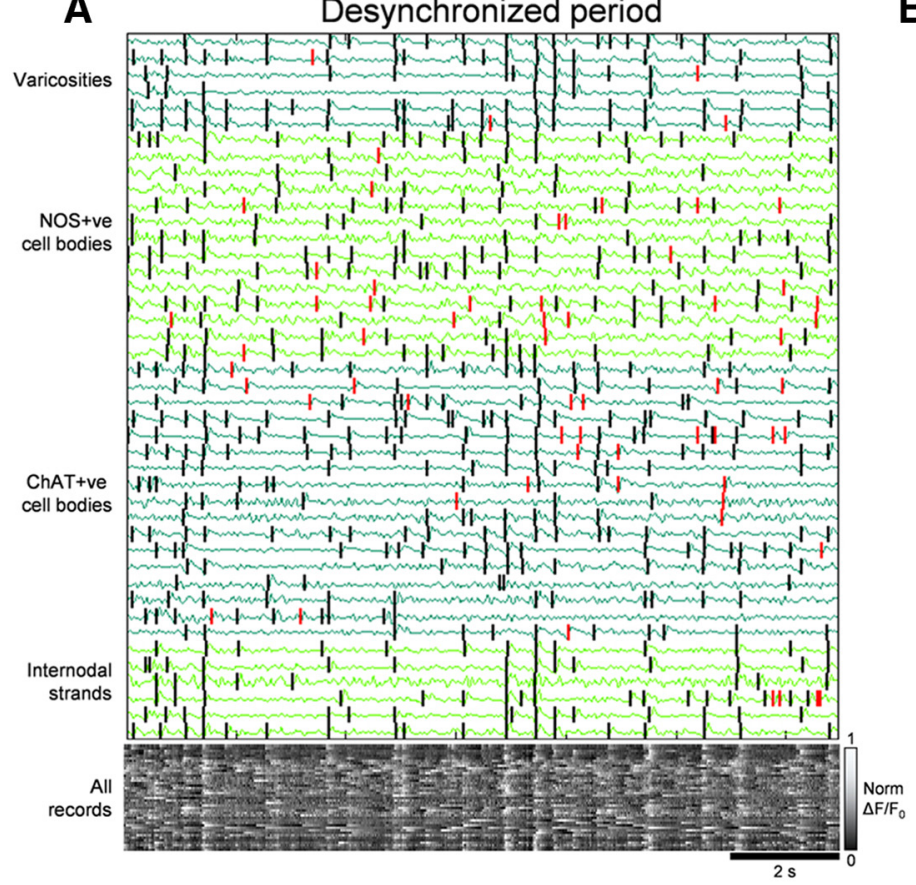

Desynchronized period
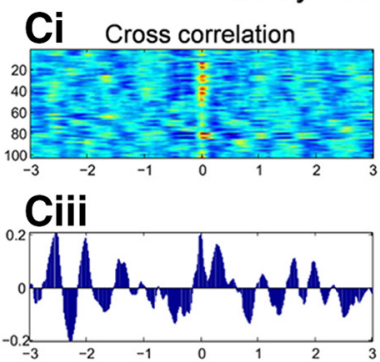

B

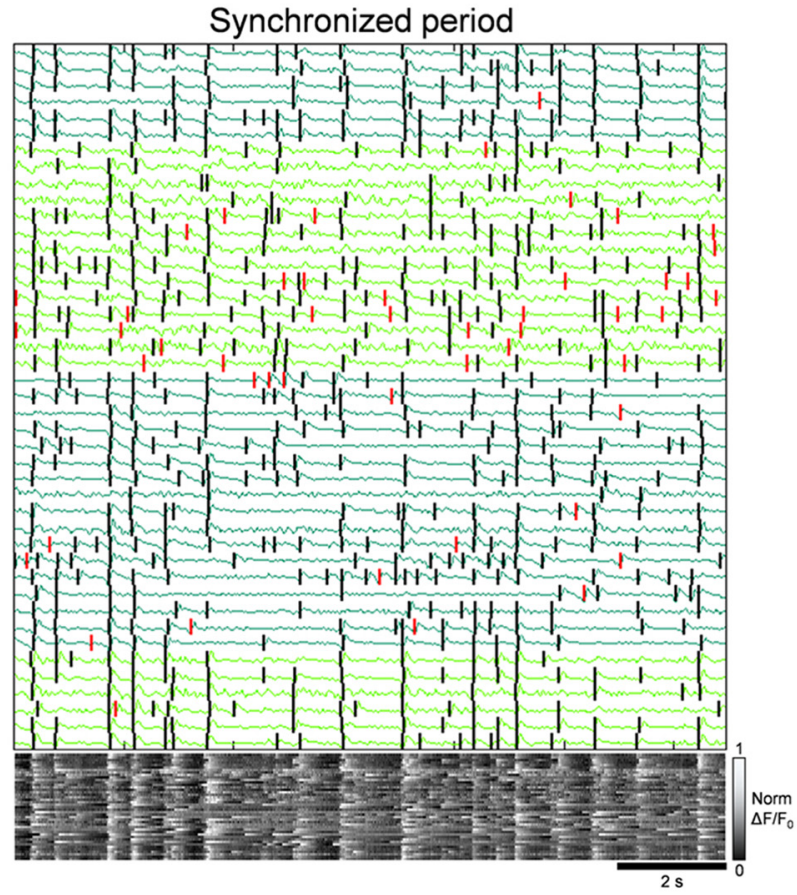

Synchronized period

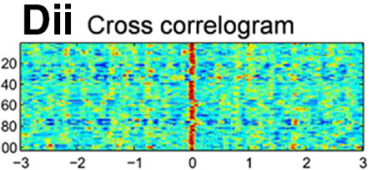

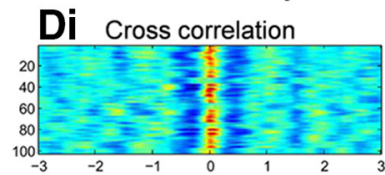

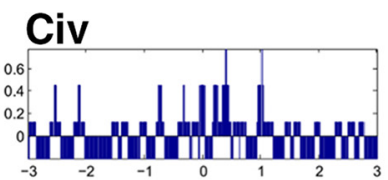

Cii Cross correlogram

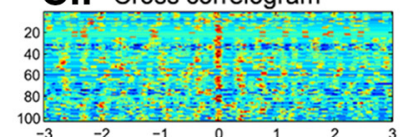

Diii

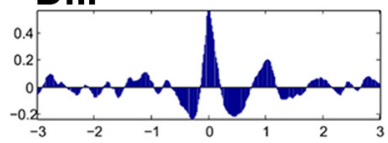

Div

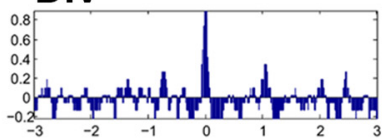

Figure 9. An example of emergence of synchronized activation in the ENS. Coincident activity patterns in recordings from different neuronal sources during desynchronized ( $\boldsymbol{A}$ ) and synchronized $(\boldsymbol{B})$ periods. The calcium activities arising from four different types of neural structures are shown in $\boldsymbol{A}$ and $\boldsymbol{B}$ : cholinergic (ChAT + ve) nerve cell bodies, nitrergic (NOS + ve) nerve cell bodies, myenteric varicosities, and internodal strands. Vertical lines denote detected calcium transients; black lines indicate transients that belong to a statistically significant pattern; red lines indicate transients that do not. In synchronized periods, significantly more neurons were involved in coincident firing patterns compared with desynchronized periods. The same records are present using a colorimetric scale below the traces. Every three lines of pixels represent one neuronal imaging site and these are shown in the same order as the traces above. Here, coincident calcium transients among imaged neurons create vertical white lines (indicating higher calcium-evoked fluorescence). The involvement of more neurons in coincident firing patterns in synchronized, compared with desynchronized period, results in the appearance of stronger, more complete white vertical lines. Ci, Ciii, Di, Diiii Cross-correlation plots where each line in Ciand Dishow the cross-correlation applied to every varicosity and ChAT + ve nerve cell body pair in plots $\boldsymbol{A}$ and $\boldsymbol{B}$ for the desynchronized and synchronized periods, respectively. Ciii, Diii, Plots give an example of one cross-correlation; two synchronized recordings will show a cross-correlation maximized at a time lag close to 0 . Here, the cross-correlation for the synchronized period, but not the desynchronized period, was maximized at a time lag of 0. Cii, Civ, Div, Div, Plots show the cross correlogram whereby each line in Cii and Dii shows the cross correlogram function applied to every varicosity and ChAT + ve nerve cell body pair in plots $\boldsymbol{A}$ and $\boldsymbol{B}$ for the desynchronized and synchronized periods, respectively. Ciii, Diii, Plots give an example of one cross correlogram; here also, two synchronized recordings will show a cross correlogram function to be maximized at a time lag close to 0 . As with cross-correlations, the cross correlogram function for the synchronized period, but not the desynchronized periods was maximized at a time lag of 0 . The increased cross-correlations and cross correlograms occurring during synchronized periods involve propagating neurogenic activity along the myenteric plexus. Figure 9-1 (available at https://doi.org/10.1523/JNEUROSCI.3489-17.2018.f9-1) shows the propagation of synchronized neurogenic activity along the myenteric plexus that occurs between two smooth muscle recordings underlying the CMMC contraction.

of junction potentials that occurred in the presence of nicardipine (Figs. 2A, B, 3A-D).

Myoelectric coherence was assessed by recording neurogenic spike bursts from two sites, ranging 1-14 mm apart (longitudinally). Coherence, but not frequency, was inversely related to separation distance (Fig. $3 E-H$ ). Beyond $7 \mathrm{~mm}$, myoelectric coherence was similar to levels seen in the presence of hexamethonium over $1 \mathrm{~mm}$. This revealed that activity in the ENS can temporally coordinate myoelectric activity over significant distances along the length of colon (many millimeters), beyond which decreases in coordination are seen.
Simultaneous neuronal imaging and electrophysiology from smooth muscle during neurogenic spike bursts

The observation that hexamethonium reduced coordinated smooth muscle activity led us to record from multiple classes of myenteric neurons simultaneously using calcium imaging (Fig. 4, Fig. 4-1 available at https://doi.org/10.1523/JNEUROSCI.348917.2018.f4-1) at the same time as smooth muscle electrophysiological recordings (Figs. 4, 5D,E). Ongoing calcium transients occurred in ChAT + ve, NOS + ve, and CGRP+ myenteric nerve cell bodies during desynchronized periods, i.e., during the intervals between neurogenic spike bursts (Figs. $4 E, 6 C, D, 7 C, 8,9 A$; 
Movie $1 ; n=12)$. The frequency of ongoing calcium transients in desynchronized periods ranged from quiescence, up to $2.6 \mathrm{~Hz}$ (average, $0.8 \pm 0.5 \mathrm{~Hz}$; Table 1 ). These activities showed relatively weak coordination among myenteric neurons ("coordination" of neural activity analyzed in detail below). However, neurogenic spike bursts (synchronized periods) were characterized by the emergence of a highly coordinated and rhythmic calcium spike pattern (Fig. 5, Fig. 5-1 available at https://doi.org/10.1523/ JNEUROSCI.3489-17.2018.f5-1). During these periods, large populations of cholinergic and nitrergic myenteric nerve cell bodies, internodal strands and varicosities were synchronously active in prolonged repetitive bursts. Neural calcium transients occurred at a frequency similar to that of smooth muscle depolarizations ( $\sim 2 \mathrm{~Hz}$; Figs. $3 A-D, 4,5 A, B, 6 E$, 7D; Table 1; Movie $2)$. The average frequency of calcium transients across all individual neurons was $1.8 \pm 0.4 \mathrm{~Hz}(n=12)$. The average frequency of the entire population of myenteric neurons active in the field of view (the ensemble or network calcium spike rate) was $1.9 \pm 0.2$ $\mathrm{Hz}(n=12)$. There was a strong correlation between calcium transients and EMG standardized global wavelet spectrum amplitudes over a range of frequencies ( $\rho \geq 0.98, n=7$; Fig. $5 C$ ). Synchronized and desynchronized periods persisted during smooth muscle action potential blockade in nicardipine ( $1 \mu \mathrm{M}, n=$ 5; Figs. 6, 7; Movie 2). However, hexamethonium always abolished temporal coordination of firing between enteric neurons $(n=5$; Movie 3), as well as neurogenic spike bursts (Fig. 1-1 available at https://doi.org/10.1523/JNEUROSCI.3489-17.2018.f1-1). The coordinated activity in synchronized periods (Fig. 6) included activities from neurons with larger nerve cell bodies (Fig. 6-1 available at https://doi.org/10.1523/JNEUROSCI.3489-17.2018. f6-1), compatible with the Dogiel type II morphology of putative intrinsic sensory neurons (Furness et al., 2004). Indeed, synchronized periods involved activity of myenteric neurons that expressed CGRP (a putative selective marker of intrinsic sensory neurons; Furness et al., 2004). Hexamethonium also abolished the $\sim 2 \mathrm{~Hz}$ pattern of activity in CGRP + ve neurons $(n=3)$.

As described above, synchronized periods persisted in nicardipine as $\sim 2 \mathrm{~Hz}$ bursts of junction potentials, in the absence of smooth muscle action potentials. As expected, the temporal activation of junction potentials occurred out of phase with respect to coordinated calcium transients in enteric neurons (Fig. 7; Movie 3). To study this further, single pulse electrical stimuli (70 $\mathrm{V}, 0.4 \mathrm{~ms}$ ) were delivered transmurally to the colon. Electrical stimulation first evoked simultaneous calcium transients in numerous enteric neurons with a latency of $50 \pm 12 \mathrm{~ms}$ (range: 28-84 ms; time from stimulus to peak of calcium transient; Fig. 7-1 available at https://doi.org/10.1523/JNEUROSCI.3489-17. 2018.f7-1). This was followed by onset of individual junction potentials in smooth muscle $218 \pm 68 \mathrm{~ms}$ from the stimulus (range: $115-300 \mathrm{~ms} ; n=4$ ). These data are consistent with the well known latency between neural activation and junction potential onset of $\sim 150 \mathrm{~ms}$ (Bennett et al., 1966; Niel et al., 1983; Spencer and Smith, 2004).

\section{Coordination of enteric neural activity underlying neurogenic contractions}

A coordinated pattern of neural activity among myenteric neurons appeared to emerge during neurogenic spike bursts. Figure 8 shows representative calcium recordings during synchronized and desynchronized periods. More neurons discharged calcium transients together during a synchronized period (Fig. 9B) than during a desynchronized period (Fig. 9A). Consistent with this, both cross correlogram and cross-correlation measures were
Table 2. The results of the cross correlogram and cross correlation analysis for each case study is given

\begin{tabular}{|c|c|c|c|c|c|c|c|c|}
\hline \multirow[b]{2}{*}{ Recording } & \multirow[b]{2}{*}{ \#Pairs } & \multicolumn{3}{|c|}{ Measure 1} & \multicolumn{3}{|c|}{ Measure 2} & \multirow{2}{*}{$\frac{\text { Measure } 3}{\text { Significant, \% }}$} \\
\hline & & \#Sync & \#Desync & Significant, \% & \#Sync & \#Desync & Significant, \% & \\
\hline 1 & 300 & 141 & 34 & 39.6 & 287 & 187 & 37.1 & 100.0 \\
\hline 2 & 1431 & 1273 & 569 & 100.0 & 1430 & 1329 & 1.1 & 100.0 \\
\hline 3 & 666 & 527 & 183 & 98.6 & 631 & 350 & 92.6 & 97.2 \\
\hline 4 & 496 & 371 & 82 & 96.4 & 381 & 295 & 10.0 & 48.8 \\
\hline 5 & 528 & 401 & 255 & 36.9 & 490 & 438 & 1.1 & 3.2 \\
\hline 6 & 1081 & 533 & 382 & 14.9 & 955 & 692 & 58.0 & 100.0 \\
\hline 7 & 703 & 412 & 129 & 80.8 & 636 & 460 & 39.1 & 100.0 \\
\hline 8 & 903 & 578 & 277 & 71.3 & 729 & 416 & 77.7 & 99.8 \\
\hline 9 & 1830 & 628 & 211 & 61.5 & 1082 & 325 & 98.2 & 88.0 \\
\hline 10 & 820 & 264 & 66 & 42.4 & 587 & 331 & 60.0 & 36.2 \\
\hline 11 & 990 & 104 & 55 & 2.2 & 695 & 129 & 99.9 & 100.0 \\
\hline 12 & 1431 & 267 & 119 & 13.7 & 1109 & 610 & 88.3 & 100.0 \\
\hline 13 & 903 & 467 & 131 & 83.9 & 847 & 690 & 24.9 & 99.2 \\
\hline 14 & 231 & 63 & 18 & 4.9 & 146 & 84 & 16.1 & 64.9 \\
\hline 15 & 1378 & 337 & 75 & 48.4 & 1080 & 1005 & 3.4 & 4.6 \\
\hline
\end{tabular}

$n=12$

The cross correlogram and cross correlation function was applied to every pair of sites; the number of pairs for each case study is given in column titled "\#Pairs". The "\#Sync" and "\#Desync" columns show the number of pairs maximized at a time lag close to 0 for the synchronized and desynchronized periods respectively. The "Significant" columns show the percentage of randomized subsets that are statistically significant with the permutation test.

maximized close to a time lag of 0 more often during synchronized periods than desynchronized periods (Fig. 9Ci-Div). Moreover, the value of cross-correlation at time lag 0 is greater during the synchronized period than a desynchronized period. These observations motivated three measures of pairwise coordination (described in Materials and Methods). The results of each measure are shown in Table $2(n=12)$. Cross correlogram and cross-correlation analyses across all different neuronal imaging sites is summarized in Table 3. These data show that the degree of coordination of activity between pairs of myenteric neurons was higher during synchronized periods. Further, more myenteric neurons were active during coincident firing patterns (Fig. 9). To quantify this, the number of neuronal structures engaged in synchronous discharge of calcium transients was analyzed by unitary event analysis (Measure 4, described in Materials and Methods; Spencer et al., 2016b). In 13 of 15 cases, more calcium transients occurred in a coincident firing pattern for the synchronized period than a desynchronized period in the same preparation $[n=$ 12; Table 4, Tab. 4-1 (available at https://doi.org/10.1523/ JNEUROSCI.3489-17.2018.t4-1) shows results using a range of coincidence firing tolerances]. This means that myenteric neurons generated synchronized calcium transients more often during neurogenic spike bursts than in the intervals between them. Together, the results show there is increased temporal coordination between enteric neurons in different ganglia during neurogenic contractions. It was clear that when synchronized neurogenic activity occurred in the myenteric plexus (Fig. 9 ), this activity propagated along the colon. This was confirmed when neuronal imaging was made between two extracellular smooth muscle recording electrodes and clear propagation of neuronal activity along the myenteric plexus was detected (Fig. 9-1 available at https://doi.org/10.1523/JNEUROSCI.348917.2018.f9-1).

\section{Smooth muscle electrical activity underlying propulsive neurogenic contractions}

Because we revealed a novel neuronal firing pattern in the ENS in flat sheet preparations of colon, we were interested to determine whether the same electrical phenomenon also occurred during the propulsion of natural content along intact tube preparations of colon. Smooth muscle electrical activity was recorded from 
Table 3. Results of the cross correlogram and cross correlation analysis comparing pairwise measures of synchrony in the synchronized and desynchronized periods

\begin{tabular}{|c|c|c|c|c|c|c|c|c|c|c|c|c|c|c|c|c|c|c|c|c|}
\hline \multirow[b]{3}{*}{ Structure } & \multirow[b]{3}{*}{ Neurochemical code } & & \multirow{2}{*}{\multicolumn{3}{|c|}{$\begin{array}{c}\begin{array}{c}\text { Internodal } \\
\text { strands }\end{array} \\
\text { Uncoded }\end{array}$}} & \multicolumn{3}{|c|}{ Varicosities } & \multicolumn{12}{|c|}{ Myenteric nerve cell bodies } \\
\hline & & & & & & & Uncod & & \multicolumn{3}{|c|}{$\begin{array}{l}\text { NOS-ve/ } \\
\text { ChAT-ve }\end{array}$} & \multicolumn{3}{|c|}{ CGRP + ve } & \multicolumn{3}{|c|}{$\mathrm{NOS}+$ ve } & \multicolumn{3}{|c|}{ ChAT + ve } \\
\hline & & & $A$ & B & $C$ & $A$ & B & $c$ & A & B & $C$ & $A$ & B & $C$ & $A$ & $B$ & $C$ & $A$ & B & $C$ \\
\hline \multirow[t]{2}{*}{ Internodal strands } & Uncoded & Synch & 85 & 99 & 0.8 & 77 & 98 & 0.7 & 56 & 87 & 0.6 & 85 & 96 & 0.7 & 49 & 88 & 0.5 & 51 & 85 & 0.5 \\
\hline & & Desynch & 56 & 94 & 0.7 & 39 & 85 & 0.5 & 27 & 69 & 0.4 & 38 & 90 & 0.5 & 18 & 61 & 0.4 & 16 & 56 & 0.3 \\
\hline \multirow[t]{2}{*}{ Varicosities } & Uncoded & Synch & & & & 65 & 92 & 0.6 & 53 & 86 & 0.5 & 67 & 95 & 0.6 & 40 & 81 & 0.5 & 40 & 76 & 0.4 \\
\hline & & Desynch & & & & 37 & 75 & 0.5 & 24 & 50 & 0.3 & 28 & 83 & 0.5 & 17 & 51 & 0.3 & 13 & 41 & 0.2 \\
\hline \multirow{8}{*}{$\begin{array}{l}\text { Myenteric nerve } \\
\text { cell bodies }\end{array}$} & $\mathrm{NOS}+\mathrm{ve} / \mathrm{ChAT}+\mathrm{ve}$ & Synch & & & & & & & 54 & 84 & 0.5 & 64 & 86 & 0.6 & 35 & 73 & 0.4 & 22 & 60 & 0.3 \\
\hline & & Desynch & & & & & & & 18 & 50 & 0.3 & 27 & 67 & 0.4 & 13 & 45 & 0.3 & 7 & 25 & 0.1 \\
\hline & CGRP + ve & Sync & & & & & & & & & & 42 & 58 & 0.5 & 49 & 91 & 0.5 & ND & ND & ND \\
\hline & & Desynch & & & & & & & & & & 11 & 56 & 0.3 & 9 & 65 & 0.4 & ND & ND & ND \\
\hline & $\mathrm{NOS}+\mathrm{ve}$ & Synch & & & & & & & & & & & & & 35 & 78 & 0.4 & 25 & 64 & 0.3 \\
\hline & & Desynch & & & & & & & & & & & & & 8 & 44 & 0.3 & 7 & 32 & 0.2 \\
\hline & ChAT + ve & Synch & & & & & & & & & & & & & & & & 34 & 69 & 0.3 \\
\hline & & Desynch & & & & & & & & & & & & & & & & 10 & 34 & 0.2 \\
\hline
\end{tabular}

Synchrony was measured between internodal strands, varicosities, and neurochemically-identified nerve cell bodies. The A columns contain the percentage of all recorded structures such that the cross correlogram is maximized close to a time lag of 0 ; the B columns contain the percentage of all recorded structures such that the cross correlation function is maximized close to a time lag of 0 ; the $C$ column contain the average value of the cross correlation function at time lag 0 over all sites. Block diagonal entries contain the results when both recordings in a pair belong to the same class (e.g., ChAT + ve versus ChAT + ve nerve cell bodies), the off-diagonals contain the results when the two recordings come from different classes (e.g., ChAT + ve versus NOS + ve nerve cell bodies). Note that ChAT/NOS and (GRP/NOS immunohistochemical combinations were used. Thus, there was no data (ND) for comparison of ChAT + ve and CGRP + ve nerve cell bodies.

Table 4. Analysis of coincident firing patterns

\begin{tabular}{lll}
\hline & \multicolumn{2}{c}{ No. of coincident calcium transients } \\
\cline { 2 - 3 } Recording & Synchronized & Desynchronized \\
\hline 1 & 68 & 40 \\
2 & 92 & 59 \\
3 & 81 & 59 \\
4 & 91 & 56 \\
5 & 88 & 70 \\
6 & 57 & 70 \\
7 & 74 & 47 \\
8 & 79 & 61 \\
9 & 59 & 30 \\
10 & 61 & 41 \\
11 & 40 & 22 \\
12 & 31 & 32 \\
13 & 67 & 49 \\
14 & 64 & 36 \\
15 & 36 & 23 \\
\hline
\end{tabular}

For each example, the maximum number of spikes in a coincident activity pattern was calculated. In 13 of 15 case studies, the maximum number of calcium transient peaks in a coincident pattern was greater during the synchronized periods. See Table 4-1 (available at https://doi.org/10.1523/JNEUROSCI.3489-17.2018.t4-1) for coincidental firing patterns that occur using different tolerances to define coincident events.

two sites in the mid-distal colon $(20$ and $30 \mathrm{~mm}$ from terminal rectum) during propulsion of 31 pellets (27 synthetic and 4 natural fecal pellets, 7 preparations, $n=7$; Fig. $10 A-C$ ). Propulsion of single fecal pellets past the electrodes was accompanied by regular action potentials at $2.0 \pm 0.1 \mathrm{~Hz}$ for a mean duration of $25.8 \pm 4.5 \mathrm{~s}(n=7)$ as the pellet passed the electrodes (Movie 4). These characteristics are indistinguishable from the neurogenic spike bursts described in flat sheet colonic preparations (Hibberd et al., 2017). In fact, the duration of the coordinated action potential spike burst did not differ between the moving pellet and the fixed sheet preparation of colon (Fig. $10 F$ ). Figure 10, $A-D$, shows a representative example. The two fine dotted lines in Figure $10 \mathrm{C}$ show the location of the fecal pellet in the proximal colon, and the migration of the single pellet toward the two electrodes located at the red and blue arrows in Figure $10 C$. The $\sim 2$ $\mathrm{Hz}$ bursts of muscle action potentials during propulsion of fecal pellets were abolished by hexamethonium $(n=5)$, suggesting that coordinated activity within the ENS was involved in coordi- nating the firing of bursts of smooth muscle action potentials at $\sim 2 \mathrm{~Hz}$.

\section{Discussion}

The present study reports the emergence of rhythmic and synchronous activity across multiple subclasses of enteric neurons and across multiple rows of ganglia, during neurogenic contractions of the large intestine. This represents a major pattern of neuronal activity in the mammalian peripheral nervous system that has not previously been identified. The synchronized ENS activity involved simultaneous activation of large populations of excitatory and inhibitory neurons, as well as putative (CGRP+ve) intrinsic sensory neurons. Synchronized ENS activity occurred at $\sim 2 \mathrm{~Hz}$ during the generation of rhythmic depolarizations in smooth muscle, which also occurred at $\sim 2 \mathrm{~Hz}$. It has not been demonstrated previously that (1) large populations of enteric neurons coordinate their firing in repetitive bursts, and (2) that electrical rhythmicity in smooth muscle can be generated by rhythmic firing in the ENS. The pattern of neuronal firing we have identified in this study directly underlies the generation of CMMCs (Balasuriya et al., 2016; Spencer et al., 2016a; Hibberd et al., 2018; Hu and Spencer, 2018).

Synchronization of activity in the nervous system is paramount for its normal functioning (Buzsaki, 2006). Spontaneous synchronization has been reported across large populations of neurons with frequencies ranging from 0.5 to 2 events $\mathrm{min}^{-1}$ occurring during early development in the cortex (Garaschuk et al., 2000) and large-scale oscillatory calcium waves in the immature cortex. Furthermore, giant synaptic potentials have been reported in the immature rat CA3 hippocampal neurons (BenAri et al., 1989), whereas in the mammalian retina, synchronous bursts of action potentials discharge in retinal ganglion cells (Meister et al., 1991) and mammalian hindbrain (Gust et al., 2003). There is also a well described development of synchronized activity in the spinal cord (O'Donovan et al., 1998). In the adult CNS, some nuclei crucial for ongoing survival retain a high degree of synchronized behavior; an example of which is in the generation of the respiratory cycle (Wittmeier et al., 2008). It is possible that the periods of synchronization and desynchronization in the ENS we revealed in this study represent a primordial 


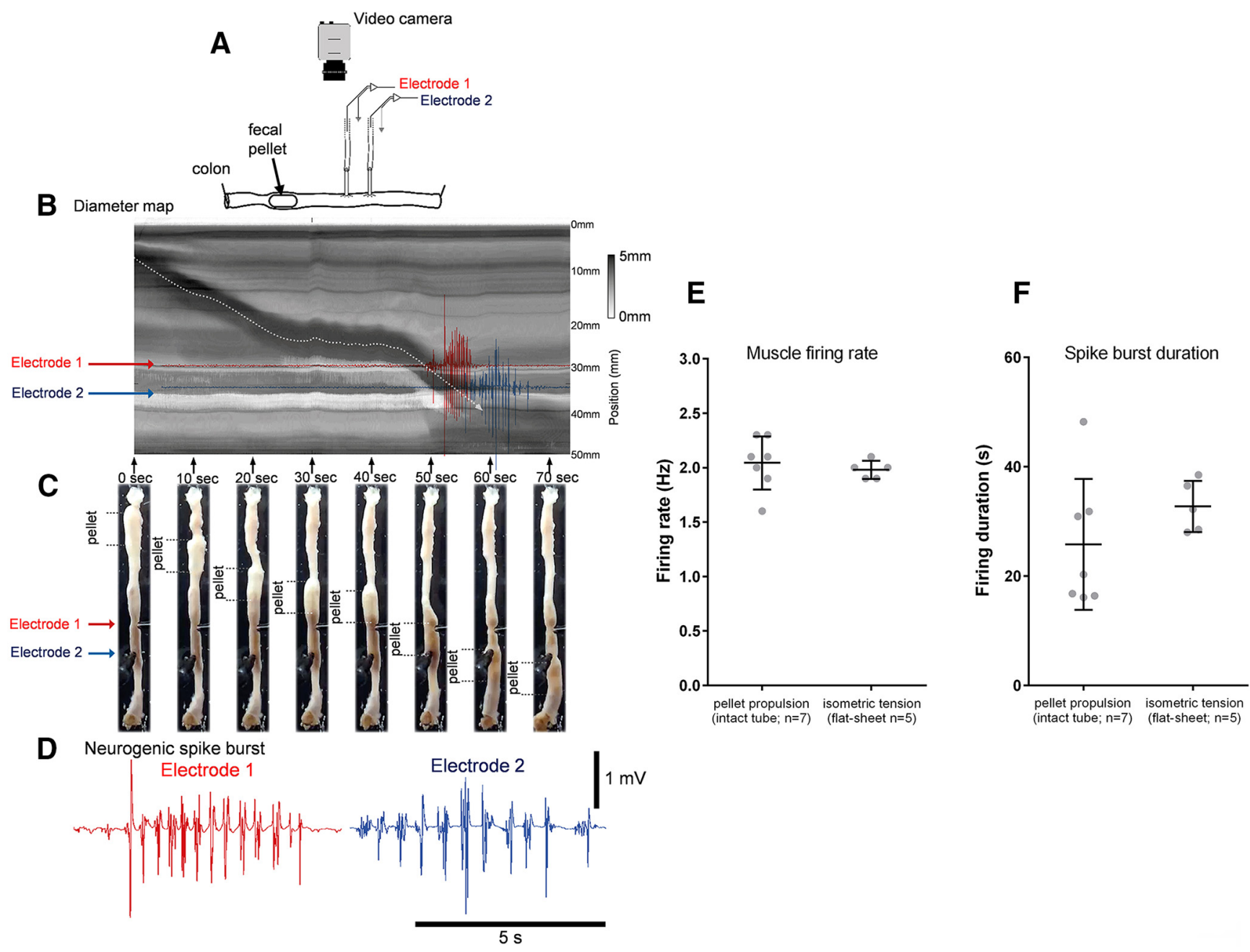

Figure 10. Electrical recordings from colonic smooth muscle during propulsion of fecal pellets in an isolated whole mouse colon. $A$, Schematic of the whole colon with natural colonic content. $A$ video camera recorded colonic wall movements and two extracellular recording electrodes recorded smooth muscle action potentials as the fecal pellets were propagated past the electrodes. $\boldsymbol{B}$, A spatiotemporal map of fecal pellet propulsion. The red and blue arrows show the relative location of the electrodes when the pellet propagates past the recording site. $C$, A series of photomicrographs as single fecal pellets propagate past the two recording sites. The red and blue arrows show the location of electrical recording sites. D, The actual discharge of action potentials in the smooth muscle in red and blue that represent the activity in the muscle as the fecal pellet is propelled past the recording. Note, the action potentials discharge in rhythmic bursts at $\sim 2 \mathrm{~Hz}$. This can also be observed in Movie 4. $\boldsymbol{E}$, The mean action potential firing rate in tubular intact preparations of whole colon versus in flat-sheet preparations of whole colon. $\boldsymbol{F}$, The action potential burst duration in the smooth muscle as content is propelled along intact tube preparations of colon compared with flat-sheet preparations.

property of the ENS, which can be considered as the primordial "brain", because rational evidence has been presented that the ENS evolved before the CNS (Furness and Stebbing, 2018).

Propagating neurogenic contractions are the predominant motor behavior of the murine large intestine. Myoelectric oscillations during this behavior at a frequency of $\sim 2 \mathrm{~Hz}$ were reported as early as 1973 (Wood, 1973). The first intracellular electrophysiological recordings from circular muscle cells of fulllength preparations of mouse colon revealed repetitive cholinergic depolarizations at $\sim 2 \mathrm{~Hz}$ during propagating neurogenic contractions (Bywater et al., 1989; Lyster et al., 1995; Spencer et al., 2005). It was hypothesized that synchronous and repetitive firing of myenteric neurons generated the rhythm and spatial coordination of inhibitory and excitatory junction potentials over distances greater than the projection lengths of motor neurons (Spencer et al., 2005; Hu and Spencer, 2018). However this was not certain because it is well known that cholinergic agonists alone can induce repetitive depolarizations in intestinal smooth muscle, when all nerve conduction is blocked (Bolton, 1971). Confirming the above hypothesis, the present study provides the first direct observation of coordinated enteric neural firing during neurogenic contractions.

Blockade of cholinergic excitatory junction potentials (EJPs) with atropine reveals repetitive inhibitory junction potentials (IJPs) alone at $\sim 2 \mathrm{~Hz}$ during neurogenic myoelectric activity (Spencer et al., 2005). Thus, it may not be surprising that nitrergic neurons in the myenteric plexus participate in ENS firing at $2 \mathrm{~Hz}$ during the neurogenic motor pattern described here. This is consistent with the observation in the present study that NOS+ve and ChAT + ve neurons were simultaneously activated at $\sim 2 \mathrm{~Hz}$. In this way, we suggest synchronized ENS firing causes pulsatile release of both excitatory and inhibitory neurotransmitter(s) simultaneously onto smooth muscle. This observation, combined with the large spatial field of ENS-coordinated myoelectric activity $(\sim 7 \mathrm{~mm})$ means that there are regions of muscle whose excitatory and inhibitory inputs are activated simultaneously during neurogenic contractions. This may be expected to interfere with smooth muscle excitation. However, IJPs are reduced, or abolished, during the rising phase of neurogenic depolarizations by a process of disinhibition, which was shown to be caused by pre- 


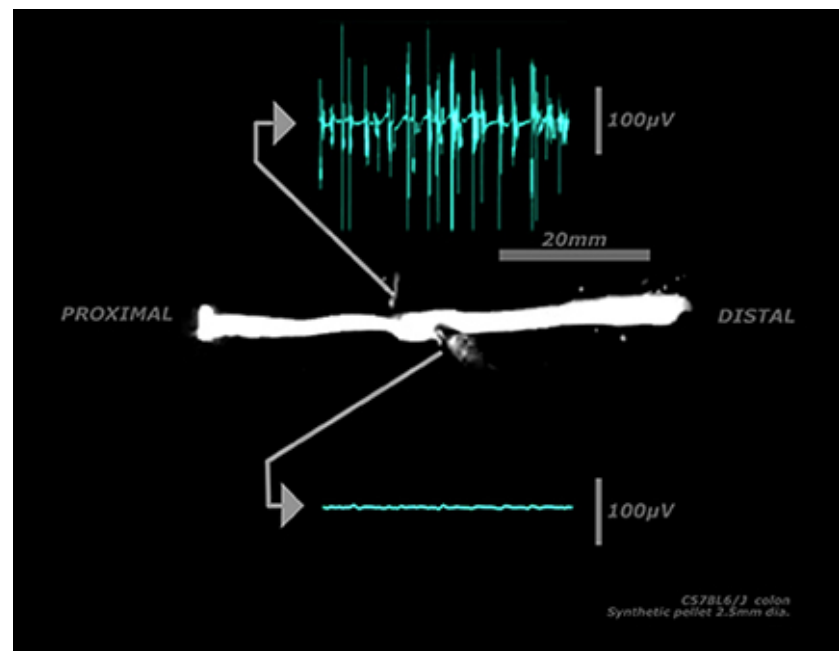

Movie 4. Simultaneous electrophysiological recordings from colonic smooth muscle during propulsion of fecal pellet along the mouse colon. Isolated whole colon is shown from a video recording made above the colon. The fecal pellet inserted into the proximal colon propagates anally. As the fecal pellet passes over the first electrode a discharge of compound action potentials occurs in bursts at $\sim 2 \mathrm{~Hz}$. As the pellet passes over the second electrode a similar burst of $2 \mathrm{~Hz}$ muscle action potentials occurs behind the fecal pellet causing contraction of the muscle that underlies propulsion.

synaptic inhibition of release of all inhibitory transmitters (both nitrergic and non-nitrergic; Spencer et al., 1998), ensuring dominance of muscle excitation. In addition, the coactivation of excitatory and inhibitory motor inputs by electrical stimuli results first in an EJP peak at $\sim 150 \mathrm{~ms}$, followed by an IJP peak $\sim 300-$ $450 \mathrm{~ms}$ (Okasora et al., 1986). This is compatible with $\sim 2 \mathrm{~Hz}$ ENS synchrony: coactivation of excitatory and inhibitory motor neurons may evoke an EJP-IJP smooth muscle membrane oscillation that is on the repolarization phase, in time for the subsequent cycle of neural input $\sim 500$ ms later.

The finding that the timing of repetitive smooth muscle depolarizations and muscle action potentials at $\sim 2 \mathrm{~Hz}$ is controlled by the coordinated firing rate of the ENS represents a vastly different mechanism from the well described myogenic slow-wave oscillations in smooth muscle (Huizinga et al., 2014). Slow waves are generated by ICCs, which are non-neuronal cells that do not require input from enteric nerves for their pacemaker activity (Huizinga et al., 1995). In contrast to myogenic slow waves, whose frequency of depolarizations is determined by ICC, the frequency of rhythmic depolarizations in smooth muscle during neurogenic contractions mirrors the synchronized firing rate of the ENS.

Unitary event analysis showed that during neurogenic contractions the different neurochemically-defined classes of myenteric neurons, including varicosities and internodal strands were activated at the same time and significantly more often. The abolition of ENS synchrony by hexamethonium suggests that bursts of fast nicotinic synaptic inputs within myenteric ganglia mediate coordinated firing of myenteric neurons at $\sim 2 \mathrm{~Hz}$. Electrophysiological recordings of myenteric neurons in small segments of mouse colon have not identified single neurons that discharge action potentials at $\sim 2 \mathrm{~Hz}$ spontaneously, or in response to imposed depolarization (Furukawa et al., 1986; Nurgali et al., 2004). Thus, whether this behavior represents an emergent property of the ENS that is not a property of individual enteric neurons, or whether a set of pacemaker neurons exists is not clear.
Of particular interest was that in addition to simultaneous activation of NOS + ve inhibitory neurons and ChAT + ve excitatory motor neurons and interneurons at $2 \mathrm{~Hz}$, putative sensory neurons in the ENS [which contain CGRP in mouse colon (Furness et al., 2004)] were also activated at $2 \mathrm{~Hz}$ (Table 3). This is compatible with the possibility that CGRP+ve myenteric neurons receive fast synaptic input in murine distal colon (Hibberd et al., 2018), as shown in some Dogiel type 2 neurons of guinea pig myenteric plexus (Grafe et al., 1979). The specific roles of these putative sensory neurons and other classes of interneurons in the generation, pacing and termination of ENS synchrony is of significant interest. Indeed, computer modeling based on the firing of guinea pig enteric sensory neurons suggests that the ENS behaves as a feed forward network whereby ". . random firing became progressively more synchronized between neurons, until the network as a whole was firing in a coordinated manner" (Thomas et al., 1999).

An important observation of this study was that the propulsion of single fecal pellets along the colon generated smooth muscle action potential firing at $\sim 2 \mathrm{~Hz}$. This suggests that regardless of whether the circumferential diameter of the colon is maintained at a fixed length (as in opened sheet preparations), or is free to change diameter as in intact tube preparations during the propulsion of content, the ENS still generates coordinated $2 \mathrm{~Hz}$ firing leading to smooth muscle action potentials at $2 \mathrm{~Hz}$.

\section{Conclusion}

Our findings demonstrate that within the peripheral nervous system, the ENS generates a rhythmic and synchronized firing pattern that causes pulsatile release of both excitatory and inhibitory neurotransmitter(s) simultaneously onto smooth muscle, over ascending excitatory and descending inhibitory spatial fields, respectively. The firing rate between bursts of smooth muscle action potentials is directly controlled by the coordinated firing of large populations of enteric neurons.

\section{References}

Balasuriya GK, Hill-Yardin EL, Gershon MD, Bornstein JC (2016) A sexually dimorphic effect of cholera toxin: rapid changes in colonic motility mediated via a 5-HT3 receptor-dependent pathway in female C57Bl/6 mice. J Physiol 594:4325-4338. CrossRef Medline

Ben-Ari Y, Cherubini E, Corradetti R, Gaiarsa JL (1989) Giant synaptic potentials in immature rat CA3 hippocampal neurones. J Physiol 416:303325. CrossRef Medline

Bennett MR, Burnstock G, Holman ME (1966) Transmission from perivascular inhibitory nerves to the smooth muscle of the guinea-pig taenia coli. J Physiol 182:527-540. CrossRef Medline

Bolton TB (1971) On the nature of the oscillations of the membrane potential (slow waves) produced by acetylcholine or carbachol in intestinal smooth muscle. J Physiol 216:403-418. CrossRef Medline

Bornstein JC, Furness JB, Kunze WA (1994) Electrophysiological characterization of myenteric neurons: how do classification schemes relate? J Auton Nerv Syst 48:1-15. CrossRef Medline

Brann L, Wood JD (1976) Motility of the large intestine of piebald-lethal mice. Am J Dig Dis 21:633-640. CrossRef Medline

Brookes SJ, Chen BN, Costa M, Humphreys CM (1999) Initiation of peristalsis by circumferential stretch of flat sheets of guinea-pig ileum. J Physiol 516:525-538. CrossRef Medline

Buzsaki G (2006) Rhythms of the brain. Oxford, UK: Oxford UP.

Bywater RA, Small RC, Taylor GS (1989) Neurogenic slow depolarizations and rapid oscillations in the membrane potential of circular muscle of mouse colon. J Physiol 413:505-519. CrossRef Medline

Carpenter BG, Hoffman A, Lee MD, GoodrichD, Betancourt B, Brubaker M, Guo J, Li P, Riddell A (2017) Stan: a probabilistic programming language. J Stat Softw 76:8217. CrossRef

Costa M, Brookes SJ (1994) The enteric nervous system. Am J Gastroenterol 89:S129-S137. Medline 
Costa M, Furness JB (1976) The peristaltic reflex: an analysis of the nerve pathways and their pharmacology. Naunyn Schmiedebergs Arch Pharmacol 294:47-60. CrossRef Medline

Costa M, Brookes SJ, Steele PA, Gibbins I, Burcher E, Kandiah CJ (1996) Neurochemical classification of myenteric neurons in the guinea-pig ileum. Neuroscience 75:949-967. CrossRef Medline

Costa M, Wiklendt L, Simpson P, Spencer NJ, Brookes SJ, Dinning PG (2015) Neuromechanical factors involved in the formation and propulsion of fecal pellets in the guinea-pig colon. Neurogastroenterol Motil 27:1466-1477. CrossRef Medline

Dickens EJ, Hirst GD, Tomita T (1999) Identification of rhythmically active cells in guinea-pig stomach. J Physiol 514:515-531. CrossRef Medline

Fida R, Lyster DJ, Bywater RA, Taylor GS (1997) Colonic migrating motor complexes (CMMCs) in the isolated mouse colon. Neurogastroenterol Motil 9:99-107. CrossRef Medline

Furness JB (2006) The enteric nervous system. Oxford, UK: Blackwell.

Furness JB, Stebbing MJ (2018) The first brain: species comparisons and evolutionary implications for the enteric and central nervous systems. Neurogastroenterol Motil 30:e13234. CrossRef Medline

Furness JB, Kunze WA, Bertrand PP, Clerc N, Bornstein JC (1998) Intrinsic primary afferent neurons of the intestine. Prog Neurobiol 54:1-18. CrossRef Medline

Furness JB, Robbins HL, Xiao J, Stebbing MJ, Nurgali K (2004) Projections and chemistry of Dogiel type II neurons in the mouse colon. Cell Tissue Res 317:1-12. CrossRef Medline

Furukawa K, Taylor GS, Bywater RA (1986) An intracellular study of myenteric neurons in the mouse colon. J Neurophysiol 55:1395-1406. CrossRef Medline

Garaschuk O, Linn J, Eilers J, Konnerth A (2000) Large-scale oscillatory calcium waves in the immature cortex. Nat Neurosci 3:452-459. CrossRef Medline

Grafe PG, Wood JD, Mayer CJ (1979) Fast excitatory postsynaptic potentials in AH (type 2) neurons of guinea pig myenteric plexus. Brain Res 163:349-352. CrossRef Medline

Gust J, Wright JJ, Pratt EB, Bosma MM (2003) Development of synchronized activity of cranial motor neurons in the segmented embryonic mouse hindbrain. J Physiol 550:123-133. CrossRef Medline

Hibberd TJ, Costa M, Travis L, Brookes SJH, Wattchow DA, Feng J, Hu H, Spencer NJ (2017) Neurogenic and myogenic patterns of electrical activity in isolated intact mouse colon. Neurogastroenterol Motil 29:1-12. CrossRef Medline

Hibberd TJ, Feng J, Luo J, Yang P, Samineni VK, Gereau RWt, Kelley N, Hu H, Spencer NJ (2018) Optogenetic Induction of Colonic Motility in Mice. Gastroenterology, in Press.

Hibberd TJ, Travis L, Wiklendt L, Costa M, Brookes SJH, Hu H, Keating DJ, Spencer NJ (2018) Synaptic activation of putative sensory neurons by hexamethonium-sensitive nerve pathways in mouse colon. Am J Physiol Gastrointest Liver Physiol 314:G53-G64. CrossRef Medline

Higgins JJ (2003) Introduction to modern nonparametric statistics. Boston, MA: Cengage Learning.

Hu H, Spencer NJ (2018) Enteric nervous system structure and neurochemistry related to function and neuropathology. In: Physiology of the gastrointestinal tract, Ed 6. (Said HM, ed), pp 629-669. Cambridge, UK: Elsevier/Academic.

Huizinga JD, Thuneberg L, Klüppel M, Malysz J, Mikkelsen HB, Bernstein A (1995) W/kit gene required for interstitial cells of Cajal and for intestinal pacemaker activity. Nature 373:347-349. CrossRef Medline

Huizinga JD, Chen JH, Zhu YF, Pawelka A, McGinn RJ, Bardakjian BL, Parsons SP, Kunze WA, Wu RY, Bercik P, Khoshdel A, Chen S, Yin S, Zhang Q, Yu Y, Gao Q, Li K, Hu X, Zarate N, Collins P, et al. (2014) The origin of segmentation motor activity in the intestine. Nat Commun 5:3326. CrossRef Medline

Lyster DJ, Bywater RA, Taylor GS (1995) Neurogenic control of myoelectric complexes in the mouse isolated colon. Gastroenterology 108:1371-1378. CrossRef Medline

Mazzuoli G, Schemann M (2009) Multifunctional rapidly adapting mechanosensitive enteric neurons (RAMEN) in the myenteric plexus of the guinea pig ileum. J Physiol 587:4681-4694. CrossRef Medline

Mazzuoli G, Schemann M (2012) Mechanosensitive enteric neurons in the myenteric plexus of the mouse intestine. PLoS One 7:e39887. CrossRef Medline

Mazzuoli-Weber G, Schemann M (2015) Mechanosensitive enteric neurons in the guinea pig gastric corpus. Front Cell Neurosci 9:430. CrossRef Medline

Meister M, Wong RO, Baylor DA, Shatz CJ (1991) Synchronous bursts of action potentials in ganglion cells of the developing mammalian retina. Science 252:939-943. CrossRef Medline

Niel JP, Bywater RA, Taylor GS (1983) Apamin-resistant post-stimulus hyperpolarization in the circular muscle of the guinea-pig ileum. J Auton Nerv Syst 9:565-569. CrossRef Medline

Nurgali K, Stebbing MJ, Furness JB (2004) Correlation of electrophysiological and morphological characteristics of enteric neurons in the mouse colon. J Comp Neurol 468:112-124. CrossRef Medline

O’Donovan MJ, Chub N, Wenner P (1998) Mechanisms of spontaneous activity in developing spinal networks. J Neurobiol 37:131-145. CrossRef Medline

Okasora T, Bywater RA, Taylor GS (1986) Projections of enteric motor neurons in the mouse distal colon. Gastroenterology 90:1964-1971. CrossRef Medline

Roberts RR, Murphy JF, Young HM, Bornstein JC (2007) Development of colonic motility in the neonatal mouse-studies using spatiotemporal maps. Am J Physiol Gastrointest Liver Physiol 292:G930-G938. CrossRef Medline

Roberts RR, Bornstein JC, Bergner AJ, Young HM (2008) Disturbances of colonic motility in mouse models of Hirschsprung's disease. Am J Physiol Gastrointest Liver Physiol 294:G996-G1008. CrossRef Medline

Sorensen J, Wiklendt L, Hibberd T, Costa M, Spencer NJ (2017) Techniques to identify and temporally correlate calcium transients between multiple regions of interest in vertebrate neural circuits. J Neurophysiol 117:885902. CrossRef Medline

Spencer NJ, Bywater RA (2002) Enteric nerve stimulation evokes a premature colonic migrating motor complex in mouse. Neurogastroenterol Motil 14:657-665. CrossRef Medline

Spencer NJ, Smith TK (2004) Mechanosensory S-neurons rather than AHneurons appear to generate a rhythmic motor pattern in guinea-pig distal colon. J Physiol 558:577-596. CrossRef Medline

Spencer NJ, Bywater RA, Taylor GS (1998) Disinhibition during myoelectric complexes in the mouse colon. J Auton Nerv Syst 71:37-47. CrossRef Medline

Spencer NJ, Hennig GW, Dickson E, Smith TK (2005) Synchronization of enteric neuronal firing during the murine colonic MMC. J Physiol 564: 829-847. CrossRef Medline

Spencer NJ, Dinning PG, Brookes SJ, Costa M (2016a) Insights into the mechanisms underlying colonic motor patterns. J Physiol 594:4099_ 4116. CrossRef Medline

Spencer NJ, Sorensen J, Travis L, Wiklendt L, Costa M, Hibberd T (2016b) Imaging activation of peptidergic spinal afferent varicosities within visceral organs using novel CGRP $\alpha$-mCherry reporter mice. Am J Physiol Gastrointest Liver Physiol 311:G880-G894. CrossRef Medline

Stan Development Team (2017) RStanArm: Bayesian applied regression modeling via Stan. R package version 2.15.3.

Thomas EA, Bertrand PP, Bornstein JC (1999) Genesis and role of coordinated firing in a feedforward network: a model study of the enteric nervous system. Neuroscience 93:1525-1537. CrossRef Medline

Torrence C, Compo GP (1998) A practical guide to wavelet analysis. Bulletin of the American Meterological Society 79:61-78. CrossRef

Wittmeier S, Song G, Duffin J, Poon CS (2008) Pacemakers handshake synchronization mechanism of mammalian respiratory rhythmogenesis. Proc Natl Acad Sci U S A 105:18000-18005. CrossRef Medline

Wood JD (1973) Electrical activity of the intestine of mice with hereditary megacolon and absence of enteric ganglion cells. Am J Dig Dis 18:477488. CrossRef Medline

Wood JD (2008) Enteric nervous system: reflexes, pattern generators and motility. Curr Opin Gastroenterol 24:149-158. CrossRef Medline

Wood JD (2012) Cellular neurophysiology of enteric neurons. In: Physiology of the gastrointestinal tract, Ed 5 (Johnson L, ed), pp 629-669. Cambridge, UK: Elsevier/Academic.

Wood JD (2016) Enteric neurobiology: discoveries and directions. Adv Exp Med Biol 891:175-191. CrossRef Medline 